Research Paper

\title{
Metabolic pathways underlying GATA6 regulating Trastuzumab resistance in Gastric Cancer cells based on untargeted metabolomics
}

\author{
Jinxia Chang ${ }^{1^{*}}$, Qiang Wang $3^{3^{*}}$, Anup Bhetuwal ${ }^{4}$, Wenhu Liu ${ }^{1,2 \bowtie}$ \\ 1. School of Basic Medical Sciences, North Sichuan Medical College, Nanchong, Sichuan 637100, China. \\ 2. School of Pharmacy, North Sichuan Medical College, Nanchong, Sichuan 637100, China. \\ 3. Department of Laboratory Medicine, Affiliated Hospital of North Sichuan Medical College; Faculty of Laboratory Medicine, Center for Translational Medicine, North \\ Sichuan Medical College, Nanchong, Sichuan 637000, China. \\ 4. Sichuan Key Laboratory of Medical Imaging and Department of Radiology, Affiliated Hospital of North Sichuan Medical College, Nanchong, Sichuan 637000, China. \\ *These authors contributed equally to this work. \\ $\triangle$ Corresponding author: Wenhu Liu, Ph.D., School of Pharmacy, North Sichuan Medical College, 55 Dongshun Rd. Nanchong, Sichuan 637100, China, E-mail: \\ wenhuliu@cqu.edu.cn.
}

(C) The author(s). This is an open access article distributed under the terms of the Creative Commons Attribution License (https://creativecommons.org/licenses/by/4.0/). See http://ivyspring.com/terms for full terms and conditions.

Received: 2020.07.11; Accepted: 2020.10.09; Published: 2020.10.23

\begin{abstract}
Trastuzumab has proven its effectiveness in gastric cancer with HER-2 gene-amplification, which has now developed resistance while the mechanism of which is not fully elucidated. Our previous studies demonstrated that the activity of GATA6 binding protein 6 (GATA6) enhanced prominently in trastuzumab resistant gastric cancer cell lines (NCI N87R and MKN45R). In the present study, we further confirmed the re-sensitization to trastuzumab and inhibition of mitochondrial functions of GATA6

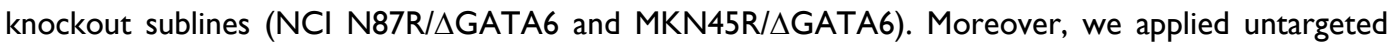
metabolomic profiling to investigate the potential roles of GATA6 in metabolism of $\mathrm{NCI} N 87 \mathrm{R}$ and MKN45R. The UPLC system coupled with Q-Exactive Focus Orbitrap mass spectrometry, multivariate in combination with univariate analysis were performed for the screening of differential metabolites between resistant cells and GATA6 knockout sublines. A total of 68 and 59 endogenous metabolites

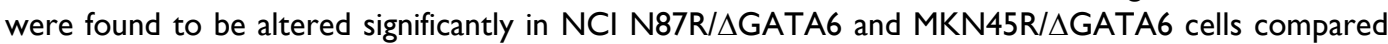
with $\mathrm{NCl} N 87 \mathrm{R}$ and MKN45R, respectively. Pathway analyses indicated disturbance of metabolic pathways after GATA6 knockout including tricarboxylic acid (TCA) cycle, glycolysis and energy-related amino acid pathways. An integrated proteomics-metabolomics revealed that sub-networks were closely related to TCA cycle, glycolysis, multiple amino acid and nucleotide metabolism. Western blot showed that TCA cycle and glycolysis-related molecules, including PKM, GLS, GLUL and LDHA, were downregulated in GATA6 knockout sublines. Taken together, these findings demonstrate that GATA6 is involved in metabolism reprogramming which might contribute to trastuzumab resistance in gastric cancer.
\end{abstract}

Key words: gastric cancer, trastuzumab resistance, GATA 6, untargeted metabolomics, TCA cycle

\section{Introduction}

Gastric cancer, one of the common malignant tumors, is characterized by sudden occurrence, rapid progression, high morbidity and mortality [1]. As part of the therapy, chemotherapy is widely applied in the treatment of gastric carcinoma, whilst drug resistance has become a primary cause of chemotherapy failure, which represents a significant challenge in the treatment of gastric cancer. Human epidermal growth factor receptor 2 (HER-2), a tyrosine kinase receptor assigned to the family of epidermal growth factor receptor (EGFR), is a crucial gastric cancer therapeutic target encoded by the c-erbB2 proto-oncogene located on chromosome 17q21[2]. HER-2 overexpression is associated with rapid tumor growth, metastasis as well as poor prognosis of gastric cancer while the activated HER-2 not only contributes to tumor 
survival signals but also facilitates reprogramming of cancer cells metabolism [3].

Trastuzumab, a monoclonal antibody targeting HER-2 receptor, has proven to be an effective agent for HER-2 positive advanced gastric cancer. However, the acquired resistance to trastuzumab impedes the curative effect and continuation of therapy. So far, the mechanisms underlying this resistance remain elusive. Hence, understanding the molecular mechanisms governing trastuzumab resistance has become of importance for seeking new therapeutic strategies.

Although the mechanisms of acquired resistance to chemotherapy in tumors are very complicated, missense mutations and metabolic reprogramming have become the most important and prioritized aspects of tumor chemotherapy failure. For instance, in pancreatic cancer disruption of glutamine metabolic pathways improves the efficacy of gemcitabine treatment [4], while HEATR1 deficiency promotes gemcitabine resistance by up-regulating Nrf2 signaling [5]. Our previous studies have demonstrated that activation of Wnt/ $\beta$-catenin/EMT pathways contributes to trastuzumab resistance in NCI N87 and MKN45 cells [6].

Transcription factor-mediated metabolic reprogramming has been implicated as a potential mechanism for drug resistance of cancer cells. GATA6, a zinc-finger transcription factor, functions as a tumor promoter or suppressor depending on the type of tumor. GATA6 overexpression has been observed in gastric cancer, breast cancer as well as in esophageal adenocarcinoma [7-9], while loss of GATA6 has been shown to be involved in malignant transformation of astrocytoma [10]. Our recent proteomic study has shown that NCI N87R and MKN45R cells display a remarkable enhancement of GATA6 activity [11], whereas NCI N87R/ $\triangle$ GATA6 and MKN45R/DGATA6 cells exhibit DNA impairment and glucose metabolism inhibition [12], suggesting that GATA6 knockout leads to an inhibition of energy metabolism. Our data therefore imply that blocking transcriptional activity of GATA6 could be an effective strategy to attenuate trastuzumab resistance in gastric cancer. Despite all of this, metabolic pathways and characteristics of GATA6 regulating trastuzumab resistance have yet to be elucidated in gastric cancer.

Metabolomics, a powerful tool to characterize complex biochemical systems, has been widely applied to life sciences fields, including illuminating the pathogenesis of cancers and mechanisms of drug resistance [13]. In this study, we employed an untargeted metabolomics based on UPLC Q-Exactive Focus mass spectrometry to investigate metabolic pathways of GATA6 regulating trastuzumab resistance in gastric cancer cells. Pathway enrichment analysis suggested perturbation in pathways of TCA cycle, multiple amino acid metabolisms related to energy, glycolysis, and nucleotide metabolisms in

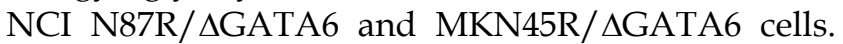
Additionally, we further analyzed the proteomic data in depth and constructed an integrated proteomicsmetabolomics network of GATA6 regulating trastuzumab resistance to illuminate the regulatory relationships between metabolic pathways and the protein expression (proteomics data were found from our previous work [12]). Our results showed that GATA6 knockout inhibited mitochondrial functions in NCI N87R and MKN45R cells, indicating that GATA6 could be a potential therapeutic target for dealing with trastuzumab resistance in gastric cancer.

\section{Materials and Methods}

\section{Chemical and reagents}

HPLC-grade acetonitrile, methanol, formic acid and ammonium acetate were supplied by CNW Inc. (Shanghai, China). 2-chloro-L-phenylalanine with a purity of $>98.5 \%$ was purchased from Heng-bai Biotech. Co. Ltd. (Shanghai, China). Deionized water was prepared from Millipore ultrapure water system (Merck Millopore, MA, USA).

\section{Cell lines and cell culture}

Trastuzumab-resistant gastric cell lines (NCI N87R, MKN45R) and GATA6 knockout sublines (NCI

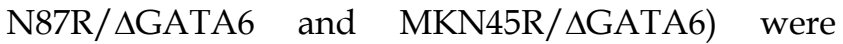
constructed and preserved in our laboratory. All cells were cultured in DMEM medium (Gibco, NY, USA) supplemented with $10 \%$ fetal bovine serum (FBS) (Gibco, NY, USA), 100 units/mL penicillin and 100 $\mu \mathrm{g} / \mathrm{mL}$ streptomycin and maintained at $37{ }^{\circ} \mathrm{C}$ in a humidified atmosphere containing 5\% $\mathrm{CO}_{2}-95 \%$ air. Cell lines were maintained in trastuzumab-containing medium $(80 \mu \mathrm{g} / \mathrm{mL})$ according to our previous procedures [6].

\section{Sample preparation for metabolome analysis}

Intracellular metabolites extractions were performed according to methods that were utilized in our previous study [14]. Briefly, cells were seeded in 6-well plates for 48-72 $\mathrm{h}$ in complete medium after which cells were harvested and counted at a density of $1 \times 10^{7}$ cells/well. Next, the cells were washed twice with pre-cold PBS, and later, the metabolites were quenched and extracted with $1000 \mu \mathrm{L}$ pre-cold mixed solvent (acetonitrile/methanol/water=2:2:1) containing $20 \mu \mathrm{L}$ of 2-chloro-L-phenylalanine as the internal standard. After this, cell pellets were collected and transferred to a microcentrifuge tube, 
homogenized for $4 \mathrm{~min}$ and sonicated for $5 \mathrm{~min}$ in ice-water bath. This was followed by incubation at 20 ${ }^{\circ} \mathrm{C}$ for $1 \mathrm{~h}$ and centrifugation at $12000 \mathrm{~g}$ for $15 \mathrm{~min}$ at 4 ${ }^{\circ} \mathrm{C}$. The supernatants were filtered through $0.22 \mu \mathrm{m}$ PTFE syringe filter. The filtrate was collected and transferred to autosampler vials for analysis by UPLC-Q Exactive Focus mass spectrometry. Quality control (QC) samples were prepared by pooling $30 \mu \mathrm{L}$ of each sample in all groups. Solvent blank and QC samples were inserted in analytical batch after every five samples to assess the stability of the detecting system.

\section{UPLC Q-Exactive Focus mass spectrometry procedure}

The metabolites were analyzed using an UPLC system (1290, Agilent) equipped with HSS T3 column (2.1 mm×100 mm, $1.8 \mu \mathrm{m}$, Waters, Milford, MA, USA). The temperature of the UPLC column oven was maintained at $35^{\circ} \mathrm{C}$ while the autosampler was set at $10^{\circ} \mathrm{C}$. For the chromatographic separation, the mobile phase in positive ionization mode (ESI+) was composed of eluent A ( $0.1 \%$ formic acid in water, v/v) and eluent $\mathrm{B}$ (acetonitrile). Likewise, in negative ionization mode (ESI-) it was composed of eluent A (5 $\mathrm{mM}$ ammonium acetate in water) and eluent B (acetonitrile). The elution gradient was set as follows: $1 \%$ B from 0 to 1 minute; $1-99 \%$ B from 1 to 8 minute; $99 \%$ B from 8 to 10 minute; $99-1 \%$ B from 10 to 10.1 minute; $1 \%$ B from 10.1 to 12.0 minute. The elution flow rate was $0.5 \mathrm{ml} / \mathrm{min}$. The injection volume was 5 $\mu \mathrm{L}$.

High-resolution mass spectrometer Q-Exactive Focus Orbitrap MS mounted on the UPLC system was used to carry out the mass spectrometry equipped with dual electrospray ionization. The operating parameters were set as follows: The spray voltage was $4.0 \mathrm{kV}$ in ESI+ and $3.6 \mathrm{kV}$ in ESI-. The temperature of capillary was $320^{\circ} \mathrm{C}$, and aux gas heater was $350{ }^{\circ} \mathrm{C}$, respectively. The aux gas and sheath gas flow rate were set at 15 arbitrary units and 30 psi, respectively. The full scan (MS1) range was 70-1000 m/z with a resolution of 70000 . The automatic gain control (AGC) target for MS acquisitions was set to $1 \times 10^{6}$ with a maximum ion injection time of $100 \mathrm{~ms}$. Subsequent MS/MS scan (MS2) was processed with a resolution of 17500 , and AGC target of $1 \times 10^{5}$ with a maximum ion injection time of $60 \mathrm{~ms}$. The dynamic exclusion time was $10 \mathrm{~s}$. The mass spectra were analyzed at normalized collisional energy (NCE). Hight-purity nitrogen was used as the nebulizing gas and collision gas for higher energy collisional dissociation. Data acquisition was performed in the mode of information-dependent acquisition (IDA).

\section{Data transformation and metabolites identification}

The acquired HPLC-MS raw files were converted to mzML files by using ProteoWizard. Subsequent data processing and analysis was accomplished using the bioinformatics program XCMS in $\mathrm{R}$ package (version 3.3.0) [15]. The parameters settings were accomplished as it was in our previous study [14]. OSI-SMMS software (version 1.0, Dalian Chem Data Solution Information Technology Co. Ltd.) was further used for peak annotation after XCMS data. The resulting generated a data that comprised of sample name, retention time (RT), mass-to-charge ratio $(\mathrm{m} / \mathrm{z})$, peak area and peak number. Metabolites were identified by matching MS/MS spectra fragmentation similarity with scores greater than or equal to 0.90 (the score range 0.00-1.00) based on self-built database containing more than 2000 endogenous metabolites.

\section{Multivariate statistical analysis and differential metabolic screening}

Peak areas were normalized to the total peak area of each chromatogram. Processing of the missing values for each sample was performed with the $80 \%$ rule while mean-centering and pareto-scaling were applied to reduce instrumental and chemical noise [16]. Then multivariate statistical analysis was performed using MetaboAnalyst (version 4.0) for unsupervised principal component analysis (PCA) to observe natural cluster trend of the differential cell lines and QC samples [17]. Subsequently, supervised orthogonal partial least square discriminant analysis (OPLS-DA) was implemented to maximize the differences of metabolic profiles between trastuzumab resistant groups and GATA6 knock out groups. The validation of all OPLS-DA models was assessed using 7-fold cross-validation and 200 permutation tests random iterations. The variable importance in the projection (VIP) plots from the OPLS-DA were applied to identify variables changed significantly in GATA6 knock out groups versus trastuzumab resistant groups. Variables with VIP values $>1.0$ were thought to be crucial which was further analyzed by independent $t$-tests. Volcano plots were accomplished by plotting the negative logarithm of $p$-value on the vertical axis (base 10) versus the logarithm of fold change (FC, base 2) on the horizontal axis. Fold change was calculated using average normalized peak areas in GATA6 knockout groups/resistant groups. SPSS 19.0 software was implemented for statistical analysis of the normalized values to determine metabolites with significant changes. Variables with VIP $>1.0, F C \geq 1.2$ or $\leq 0.83$ and $p$ value $<0.05$ were regarded as differentially altered metabolites. Data 
were displayed as mean \pm SEM and presented by GraphPad Prism 8.0.1 (GraphPad Prism, Inc. San Diego, USA).

\section{Integrated analysis of metabolomics and proteomics}

MetaboAnalyst (version 4.0) was applied to explore biological functions of differential metabolites which set the cut-off of pathway impact value from the topology analysis to 0.1 as according to previous methods [17]. The Kyoto Encyclopedia of Genes and Genomes database (KEGG, version 89.1) was explored to investigate the disturbed metabolic pathways. OmicsNet database (version 1.0) was utilized to acquire the network analysis and molecular interactions with the standard setting [18]. Cytoscape software (version 3.7.2) was applied to visualize the network models from differential metabolites and regulatory target genes.

\section{Western blotting assay}

Western blot analysis was performed according to protocols described recently [6]. Briefly, cells from different groups were lysed using RIPA lysis buffer (50 mM Tris, $150 \mathrm{mM} \mathrm{NaCl}, 1 \%$ Triton X-100, 1\% sodium deoxycholate, $0.1 \%$ sodium dodecyl sulfate, Beyotime, China) containing 1\% protease inhibitor. Lysates were centrifuged for $15 \mathrm{~min}$ at $4{ }^{\circ} \mathrm{C}(12000 \mathrm{~g})$, after which the supernatant was collected for further use. Proteins concentration were quantified with the Broadford assay kit (CWBIO, China). Equal amounts $(20 \mu \mathrm{g})$ of proteins were denatured by heating and separated by SDS-PAGE, followed by transfer to nitrocellulose membranes which were later incubated with designated primary antibodies against PKM (Abcam, \#38237, UK), GLS2 (Abcam, \#113509, UK), GLUL (Abcam, \#49873, UK), LDHA (Abcam, \#125683, UK) and $\beta$-actin (Cell Signaling, \#5176, USA) (dilution $1: 1000)$ respectively at $4{ }^{\circ} \mathrm{C}$ overnight after blocking with $5 \%$ skimmed milk. Thereafter, membranes were incubated with suitable secondary antibodies (ZSGBBio, China) (dilution 1:3000) at room temperature. Finally, chemiluminescence signals were visualized using an enhanced chemiluminescence reagent (CWBIO, China). The grey values of these signals were measured using Image $\mathrm{J}$ and histograms were plotted using GraphPad Prism 8.0.1.

\section{Cell viability assays}

Cell viability was assessed using CCK-8 kit according to previous protocols [6]. Cells were seeded in a 96-well plate at $4 \times 10^{3}$ cells / well and incubated for $24 \mathrm{~h}$. The cells were then treated with a range of indicated concentrate of trastuzumab $(0,20,40,80$, 160, 320 and $640 \mu \mathrm{g} / \mathrm{mL}$ ) for $72 \mathrm{~h}$. Absorbance was detected at $450 \mathrm{~nm}$ with a microplate reader (Bio-Rad,
Hercules, CA, USA). All data were represented based on three independent experiments.

\section{Mitochondria isolation and purification}

Mitochondria isolation were executed using a Cell Mitochondria Isolation Kit (Beyotime, \#C3601, China) according to the manufacturer's instructions. Briefly, $5 \times 10^{7}$ cells were prepared and washed twice with pre-cold PBS and centrifuged at $800 \mathrm{~g}$ for $5 \mathrm{~min}$ at $4{ }^{\circ} \mathrm{C}$. Thereafter, cells were homogenized 50 times using a homogenizer with the mitochondria isolation buffer supplemented with $1 \mathrm{mM}$ protease inhibitor. The cell homogenates were then centrifuged at $1000 \mathrm{~g}$ for $10 \mathrm{~min}$ at $4{ }^{\circ} \mathrm{C}$. The supernatant was transferred to 1.5-mL tubes and further centrifuged at $3500 \mathrm{~g}$ for 10 min at $4{ }^{\circ} \mathrm{C}$. The mitochondria pellet was resuspended with the mitochondria storage buffer for further analysis.

\section{Detection of mitochondrial membrane potential}

Mitochondrial membrane potential was determined using Mitochondrial Membrane Potential Assay Kit with JC-1 (Beyotime, \#C2006, China). Purified mitochondria were mixed with JC-1 buffer $(1 \times)$. Fluorescence intensity was detected with Multi-Mode Reader (BioTek) at an excitation of 485 $\mathrm{nm}$ and emission wavelength of $535 \mathrm{~nm}$ for JC-1 monomers and an excitation of $535 \mathrm{~nm}$ and emission wavelength of $595 \mathrm{~nm}$ for J-aggregates. The mitochondrial membrane potential was presented as the ratio of J-aggregates to monomers.

\section{Detection of the cellular adenosine triphosphate in mitochondria}

ATP levels in mitochondria were detected using an ATP Assay Kit (Beyotime, \#S0027, China) according to the manufactures' instructions. $100 \mu \mathrm{L}$ ATP working reagent was added to a 96-well plate and put at room temperature for $5 \mathrm{~min}$ and then added with $20 \mu \mathrm{L}$ of purified mitochondria per-well. The ATP levels were tested using a Multi-mode reader (BioTek) and calculated according to the standard ATP curve.

\section{Results}

\section{GATA6 knockout re-sensitized resistant cells to trastuzumab}

To investigate if GATA6 knockout decreased trastuzumab resistance in gastric cancer cells, NCI N87R, NCI N87R/ $\triangle$ GATA6, MKN45R and MKN45R/ $\triangle$ GATA6 cells were cultured under indicated concentrations of trastuzumab for $72 \mathrm{~h}$. The results showed trastuzumab inhibited viability of NCI N87R, NCI N87R/ $\triangle$ GATA6, MKN45R and MKN45R/ 
$\triangle$ GATA6 cells in a dose-dependent manner, while the more distinct inhibitory effects were observed in NCI N87R/ $\triangle$ GATA6 and MKN45R/ $\Delta$ GATA6. It reached significance from a statistical standpoint at $80 \mu \mathrm{g} / \mathrm{mL}$ with cell viability of $90.25 \%$ and $81.62 \%$ in NCI N87R and NCI N87R/ $\triangle$ GATA6, and $160 \mu \mathrm{g} / \mathrm{mL}$ with cell viability of $88.98 \%$ and $82.37 \%$ in MKN45R and MKN45R/ $\triangle$ GATA6 cells, respectively (Fig. 1A, B). These results demonstrated that GATA6 knockout re-sensitized resistant cells to trastuzumab.

\section{GATA6 knockout inhibited mitochondrial function of trastuzumab resistant gastric cancer cells}

We also detected the MMP and ATP levels between trastuzumab resistant groups and GATA6 knockout groups, respectively. The results displayed a relatively lower levels of ATP and MMP in GATA6 knockout groups compared to control groups (Fig. 1C, D), suggesting that knockout of GATA6 inhibited mitochondrial function and affected energy metabolism of trastuzumab resistant cells.

\section{Quality control analysis of mass spectrometric data}

To evaluate the quality of the mass spectrometric data, pooled QC samples and standard compound (2-chloro-L-phenylalanine) were applied to monitor the stability of the LC/MS systems and control the reproducibility of the sample treatment procedures. The results indicated that QC samples were clustered tightly in PCA scatter plot (Fig. 2A, E, I, M) with relative standard deviations (RSD\%) of peak areas being $3.24 \%$ in positive ionization mode (ESI+) and $4.79 \%$ in negative ionization mode (ESI-), respectively (Table S1). PCA-X one-dimensional score plots displayed good reproducibility in trastuzumab resistant groups and GATA6 knockout groups with both positive and negative ion ionization modes within \pm 2 standard deviation (Std), respectively (Fig. S1). Moreover, the high correlation $(r>0.90)$ of peak areas from QC samples demonstrated a good reproducibility (Fig. S2). Clearly, these results indicated a satisfactory stability of the data acquisition system.

\section{Metabolomics profiling of trastuzumab resistant gastric cancer cells and trastuzumab resistant gastric cancer cells with GATA6 knockout}

To disclose the metabolic behaviors between trastuzumab resistant gastric cancer cells with GATA6 knockout and trastuzumab resistant cells, an untargeted metabolomics-based strategy was applied to profile the metabolites in ESI+ and ESI-, respectively. Total of 3990 and 3894 features were detected from NCI N87R/ $\triangle$ GATA6, NCI N87R and MKN45R/ $\triangle$ GATA6, MKN45R cells in ESI+, whereas
A

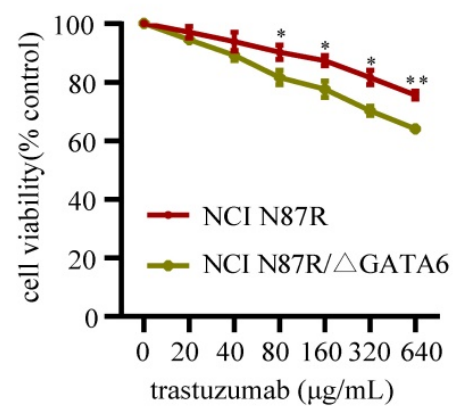

$\mathrm{C}$

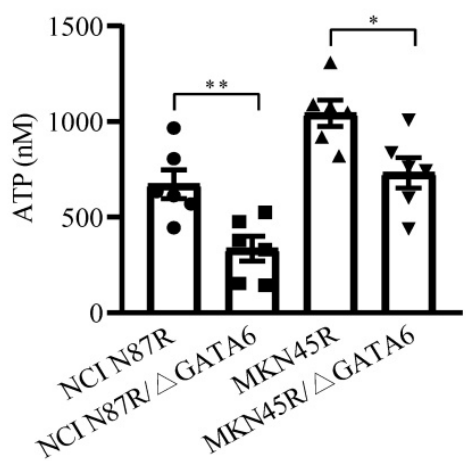

B

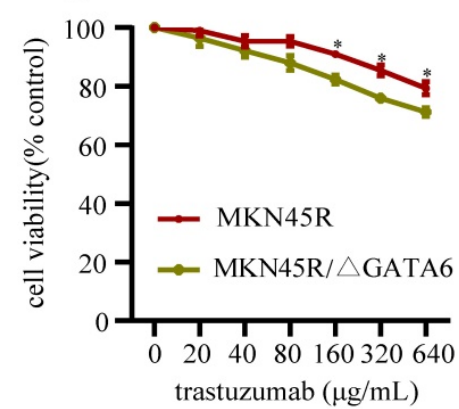

$\mathrm{D}$

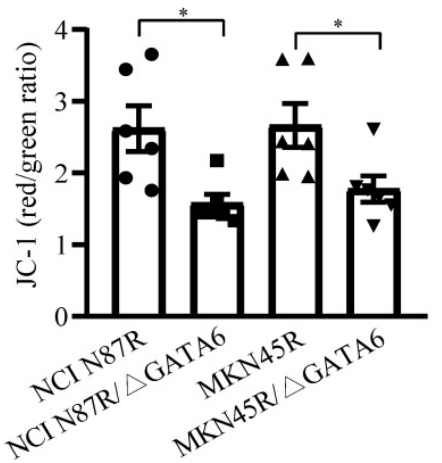

Figure 1. Detection of cell viability and mitochondrial function. (A and B) Cells were incubated with different concentrations of trastuzumab for $72 \mathrm{~h}$, and then cell viability was measured by CCK-8 kit. (C) ATP levels were tested using ATP assay Kit. (D) MMPs were detected using Mitochondrial Membrane Potential Assay Kit with JC-1. Six independent biological replicates are shown as mean \pm SEM. ${ }^{*} p<0.05,{ }^{*} p<0.01$ vs. controls. 
4335 and 4216 features were obtained in ESI-, respectively. These features matched successfully with 73 (ESI+) and 68 (ESI-) metabolites in NCI N87R/ $\triangle$ GATA6 and NCI N87R cells, along with 66 (ESI+) and 59 (ESI-) metabolites in MNK45R/ $\triangle$ GATA6 and MKN45R cells and associated with MS2 scores more than 0.90 (score range: 0.00-1.00). PCA was performed at first to discern the presence of variable differences in mass spectral profiles between trastuzumab resistant groups and GATA6 knockout groups in both ion modes. The apparent differences indicated intrinsic variations in trastuzumab resistant cells with GATA6 knockout versus trastuzumab resistant cells (Fig. 2A, E, I, M). Next, a supervised OPLS-DA was employed to distinguish the differences further as evident from the score plots which showed notable separation between different groups without overlap (Fig. 2B, F, J, N). The results of the OPLS-DA modes were explained by variance R2X (0.839 and 0.891 for ESI+, 0.876 and 0.923 for ESI- in NCI N87R/ $\triangle$ GATA6 and MKN45R/ $\triangle$ GATA6, respectively), R2Y (0.983 and 0.916 for ESI+, 0.990 and 0.969 for ESI- in NCI N87R/ $\triangle$ GATA6 and MKN45R/ $\triangle$ GATA6, respectively), and predicted variance Q2 (0.944 and 0.925 for ESI+, 0.962 and 0.946 for ESI- in

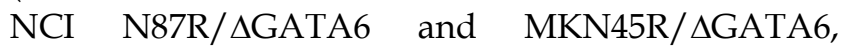
respectively). This indicated the classification models had a good explanatory and predictive ability for all models. To prevent model overfitting, the validity of OPLS-DA models was further analyzed using 200 iterations permutation tests with intercepts values $\mathrm{R} 2=0.836, \mathrm{Q} 2=-0.454$ (for ESI+), and $\mathrm{R} 2=0.787, \mathrm{Q} 2=-$ 0.484 (for ESI-) in NCI N87R/ $\Delta$ GATA6 cells (Fig. 2C and G), respectively. Similarly validity in MKN45R/ $\triangle$ GATA6 cells were evaluated with intercepts values $\mathrm{R} 2=0.789, \mathrm{Q} 2=-0.444$ (for ESI + ), and $\mathrm{R} 2=0.795, \mathrm{Q} 2=-$ 0.551 (for ESI-), respectively (Fig. $2 \mathrm{~K}$ and O). These results revealed that OPLS-DA model was reliable and not over-fitted. Next, a heatmap was applied to visualize the whole metabolome comparison of both the groups, which indicated a significant change of the metabolome (Fig. S3).

\section{Identification of differential metabolites}

Based on the OPLS-DA models, VIP-plots were explored to analyze critical variables that contributed to distinguishing the metabolome between GATA6 knockout groups and resistant groups in both ion modes (Fig. 2D, H, L, P). Those variables with VIP value $>1$ were considered as potential candidates of significance. Subsequently, volcano plots were used to screen differential metabolite abundances against the corresponding $p$ value obtained from unpairedsample $t$-test (Fig. 4A-D). Differential metabolites were confirmed by matching retention time and MS2 fragmentation patterns (MS2 scores $\geq 0.90$ ) from self-built database. Compared with NCI N87R and MKN45R cells, the levels of 11 and 22 metabolites displayed a more than 1.2 fold increase in NCI N87R/ $\triangle$ GATA6 and MKN45R/ $\triangle$ GATA6 cells (VIP>1 and $p<0.05)$, respectively. Similarly, 57 and 37 metabolites showed a less than 0.83 fold decrease in NCI N87R/ $\triangle$ GATA6 and MKN45R/ $\Delta$ GATA6 cells (VIP>1, $p<0.05$ ), respectively (Fig. $3 \mathrm{~A}$, Tables 1 \& 2). A hierarchical cluster visualization of differential metabolites indicated that majority of metabolites sharply decreased in GATA6 knock out groups versus resistant groups (Fig. 3B, C). From the Venn diagram, it was determined that 47 metabolites overlapped in NCI N87R/ $\triangle$ GATA6 and MKN45R/ $\Delta$ GATA6 cells (Table S2), however, 22 identified metabolites exclusively were of NCI N87R and NCI N87R/ $\triangle$ GATA6 cells (Fig. 3D, Table S3), whereas 12 metabolites were exclusive of MKN45R and MKN45R/ $\triangle$ GATA6 cells (Fig. 3D, Table S4). These metabolites were further classified according to their properties, including amino acids, amines, organic acids, nucleotides, cofactors, carbohydrates and others, of which organic acid, amino acid and nucleotides accounted for more than half of all identified metabolites (Fig. 3E, F).

Table 1. Significantly changed metabolites in NCI N87R/AGATA6 compared with NCI N87R cells

\begin{tabular}{|c|c|c|c|c|c|c|c|c|c|c|}
\hline No. & Name & Adduct & Observed mass & Exact mass & Mass error (ppm) & $\mathrm{RT}(\mathrm{s})^{\mathrm{a}}$ & VIP value ${ }^{b}$ & FCc & $p$ value & ESId \\
\hline 1 & Proline & {$[\mathrm{M}+\mathrm{H}]+$} & 116.0705 & 115.0633 & -6.9 & 35.42 & 4.58 & 0.59 & $5.74 \times 10^{-5}$ & + \\
\hline 2 & Cellobiose & {$[\mathrm{M}+\mathrm{Na}]+$} & 365.1047 & 342.1162 & -1.5 & 33.79 & 1.03 & 8.39 & $2.76 \times 10^{-14}$ & + \\
\hline 3 & Choline & {$[\mathrm{M}]+$} & 104.1069 & 104.1075 & -5.8 & 32.47 & 2.97 & 0.40 & $5.87 \times 10^{-11}$ & + \\
\hline 4 & 5'-Methylthioadenosine & {$[\mathrm{M}+\mathrm{H}]+$} & 298.0963 & 297.0896 & -4.4 & 35.84 & 5.21 & 0.62 & $3.67 \times 10^{-5}$ & + \\
\hline 5 & Adenine & {$[\mathrm{M}+\mathrm{H}]+$} & 136.0617 & 135.0545 & -5.9 & 44.45 & 1.36 & 0.09 & $2.69 \times 10^{-7}$ & + \\
\hline 6 & Creatine & {$[\mathrm{M}+\mathrm{H}]+$} & 132.0767 & 131.0695 & -6.1 & 35.70 & 2.09 & 0.71 & $1.36 \times 10^{-3}$ & + \\
\hline 7 & Thiamine & {$[\mathrm{M}]+$} & 265.1113 & 265.1123 & -3.8 & 36.48 & 11.73 & 0.50 & $2.51 \times 10^{-8}$ & + \\
\hline 8 & Guanosine & {$[\mathrm{M}+\mathrm{H}]+$} & 284.0984 & 283.0917 & -4.6 & 135.82 & 1.05 & 0.21 & $1.61 \times 10^{-10}$ & + \\
\hline 9 & Valine & {$[\mathrm{M}-\mathrm{H}]-$} & 116.0719 & 117.0789 & 8.5 & 35.95 & 1.75 & 0.56 & $6.28 \times 10^{-6}$ & - \\
\hline 10 & Lysine & {$[\mathrm{M}+\mathrm{H}]+$} & 147.1127 & 146.1055 & -5.4 & 28.71 & 1.35 & 0.58 & $3.44 \times 10^{-8}$ & + \\
\hline 11 & Citrulline & {$[\mathrm{M}-\mathrm{H}]-$} & 174.0886 & 175.0957 & 5.1 & 31.66 & 2.75 & 0.44 & $4.21 \times 10^{-9}$ & - \\
\hline 12 & Pyroglutamic acid & {$[\mathrm{M}+\mathrm{H}]+$} & 130.0498 & 129.0425 & -5.4 & 91.95 & 1.29 & 0.24 & $6.72 \times 10^{-11}$ & + \\
\hline
\end{tabular}




\begin{tabular}{|c|c|c|c|c|c|c|c|c|c|c|}
\hline No. & Name & Adduct & Observed mass & Exact mass & Mass error (ppm) & $\mathrm{RT}(\mathrm{s})^{\mathrm{a}}$ & VIP value ${ }^{b}$ & $\mathrm{FCc}$ & $p$ value & ESId \\
\hline 13 & Histidine & {$[\mathrm{M}+\mathrm{H}]+$} & 156.0767 & 155.0695 & -5.1 & 30.32 & 1.01 & 0.39 & $6.43 \times 10^{-10}$ & + \\
\hline 14 & GMP & {$[\mathrm{M}+\mathrm{H}]+$} & 364.0644 & 363.0579 & -4.1 & 56.79 & 1.53 & 2.84 & $3.11 \times 10^{-9}$ & + \\
\hline 15 & Glutamic acid & {$[\mathrm{M}+\mathrm{H}]+$} & 148.0603 & 147.0532 & -6.1 & 32.34 & 1.57 & 0.51 & $3.97 \times 10^{-6}$ & + \\
\hline 16 & Taurine & {$[\mathrm{M}+\mathrm{H}]+$} & 126.0219 & 125.0147 & -6.4 & 31.12 & 1.39 & 0.58 & $1.16 \times 10^{-6}$ & + \\
\hline 17 & Citicoline & {$[\mathrm{M}+\mathrm{H}]+$} & 489.1135 & 488.1073 & -3.6 & 35.33 & 1.09 & 0.20 & $1.49 \times 10^{-8}$ & + \\
\hline 18 & Pantothenic acid & {$[\mathrm{M}-\mathrm{H}]-$} & 218.1036 & 219.1107 & 4.1 & 44.69 & 2.98 & 0.11 & $1.29 \times 10^{-7}$ & - \\
\hline 19 & Niacinamide & {$[\mathrm{M}+\mathrm{H}]+$} & 123.0552 & 122.0480 & -6.5 & 40.89 & 1.44 & 0.74 & $6.37 \times 10^{-3}$ & + \\
\hline 20 & Acetylcarnitine & {$[\mathrm{M}+\mathrm{H}]+$} & 204.1229 & 203.1157 & -3.9 & 56.96 & 2.19 & 0.76 & $3.95 \times 10^{-2}$ & + \\
\hline 21 & Phosphorylcholine & {$[\mathrm{M}]+$} & 184.0732 & 184.0738 & -3.2 & 56.18 & 1.89 & 0.57 & $9.40 \times 10^{-4}$ & + \\
\hline 22 & Glycerophosphocholine & {$[\mathrm{M}+\mathrm{H}]+$} & 258.1098 & 257.1028 & -3.9 & 31.77 & 1.17 & 0.49 & $2.22 \times 10^{-6}$ & + \\
\hline 23 & Palmitoylethanolamide & {$[\mathrm{M}+\mathrm{H}]+$} & 300.2892 & 299.2824 & -4.0 & 480.69 & 4.38 & 0.33 & $4.47 \times 10^{-8}$ & + \\
\hline 24 & Carnitine & {$[\mathrm{M}+\mathrm{H}]+$} & 162.1123 & 161.1052 & -5.6 & 34.33 & 1.12 & 0.76 & $1.41 \times 10^{-2}$ & + \\
\hline 25 & S-Adenosylmethionine & {$[\mathrm{M}]+$} & 399.1437 & 399.1451 & -3.5 & 36.27 & 1.19 & 0.53 & $1.56 \times 10^{-4}$ & + \\
\hline 26 & Tyrosine & {$[\mathrm{M}-\mathrm{H}]-$} & 180.0669 & 181.0739 & 5.5 & 56.96 & 2.17 & 0.42 & $7.40 \times 10^{-7}$ & - \\
\hline 27 & Propionylcarnitine & {$[\mathrm{M}+\mathrm{H}]+$} & 218.1384 & 217.1314 & -4.6 & 133.78 & 1.24 & 0.32 & $4.16 \times 10^{-7}$ & + \\
\hline 28 & Cysteinylglycine & {$[\mathrm{M}+\mathrm{H}]+$} & 179.0484 & 178.0412 & -4.5 & 44.91 & 1.51 & 0.32 & $2.55 \times 10^{-5}$ & + \\
\hline 29 & NAD & {$[\mathrm{M}+\mathrm{H}]+$} & 664.1149 & 663.1091 & -3.3 & 56.79 & 2.40 & 0.46 & $3.45 \times 10^{-6}$ & + \\
\hline 30 & 2-Methylbutyroylcarnitine & {$[\mathrm{M}+\mathrm{H}]+$} & 246.1697 & 245.1627 & -4.1 & 196.15 & 1.63 & 0.64 & $1.82 \times 10^{-3}$ & + \\
\hline 31 & Guanine & {$[\mathrm{M}+\mathrm{H}]+$} & 152.0566 & 151.0494 & -5.3 & 135.84 & 2.84 & 0.22 & $9.57 \times 10^{-11}$ & + \\
\hline 32 & Palmitoylcarnitine & {$[\mathrm{M}+\mathrm{H}]+$} & 400.3412 & 399.3349 & -4.2 & 613.81 & 5.53 & 0.65 & $2.32 \times 10^{-2}$ & + \\
\hline 33 & N-Acetyl-L-aspartic acid & {$[\mathrm{M}-\mathrm{H}]-$} & 174.0409 & 175.0481 & 4.6 & 25.90 & 1.30 & 0.42 & $2.25 \times 10^{-9}$ & - \\
\hline 34 & Tetradecanoylcarnitine & {$[\mathrm{M}+\mathrm{H}]+$} & 372.3101 & 371.3036 & -4.0 & 541.29 & 2.47 & 0.31 & $4.31 \times 10^{-9}$ & + \\
\hline 35 & 4-Trimethylammoniobutanoic acid & {$[\mathrm{M}+\mathrm{H}]+$} & 146.1175 & 145.1103 & -5.5 & 177.67 & 1.27 & 0.78 & $2.43 \times 10^{-5}$ & + \\
\hline 36 & Glutamine & {$[\mathrm{M}+\mathrm{H}]+$} & 147.0763 & 146.0691 & -5.5 & 31.57 & 4.17 & 0.10 & $1.60 \times 10^{-14}$ & + \\
\hline 37 & Adenosine & {$[\mathrm{M}+\mathrm{H}]+$} & 268.1036 & 267.0968 & -4.5 & 135.80 & 1.02 & 0.02 & $2.62 \times 10^{-11}$ & + \\
\hline 38 & Sphinganine & {$[\mathrm{M}+\mathrm{H}]+$} & 302.3047 & 301.2980 & -4.3 & 534.68 & 1.97 & 2.94 & $4.67 \times 10^{-7}$ & + \\
\hline 39 & Elaidic carnitine & {$[\mathrm{M}+\mathrm{H}]+$} & 426.3569 & 425.3505 & -3.8 & 645.00 & 1.49 & 0.79 & $4.41 \times 10^{-4}$ & + \\
\hline 40 & Xanthine & {$[\mathrm{M}-\mathrm{H}]-$} & 151.0263 & 152.0334 & 5.9 & 58.40 & 1.64 & 0.34 & $1.79 \times 10^{-3}$ & - \\
\hline 41 & Stearic acid & {$[\mathrm{M}-\mathrm{H}]-$} & 283.2645 & 284.2715 & 3.5 & 547.56 & 2.97 & 0.64 & $4.31 \times 10^{-6}$ & - \\
\hline 42 & Phosphoenolpyruvic acid & {$[\mathrm{M}-\mathrm{H}]-$} & 166.9752 & 167.9823 & 5.4 & 24.85 & 1.30 & 0.18 & $1.63 \times 10^{-10}$ & - \\
\hline 43 & Citric/Isocitric acid & {$[\mathrm{M}-\mathrm{H}]-$} & 191.0199 & 192.0270 & 4.7 & 25.72 & 2.46 & 0.64 & $1.75 \times 10^{-3}$ & - \\
\hline 44 & Malic acid & {$[\mathrm{M}-\mathrm{H}]-$} & 133.0144 & 134.0215 & 6.7 & 25.33 & 2.22 & 0.59 & $1.44 \times 10^{-6}$ & - \\
\hline 45 & Glutathione & {$[\mathrm{M}-\mathrm{H}]-$} & 306.0745 & 307.0838 & -4.2 & 26.62 & 1.25 & 0.69 & $2.02 \times 10^{-2}$ & - \\
\hline 46 & Palmitoleic acid & {$[\mathrm{M}-\mathrm{H}]-$} & 253.2176 & 254.2245 & 4.3 & 452.15 & 2.16 & 1.62 & $5.51 \times 10^{-5}$ & - \\
\hline 47 & Glyceric acid & {$[\mathrm{M}-\mathrm{H}]-$} & 105.0194 & 106.0266 & 7.5 & 27.38 & 1.10 & 0.09 & $3.80 \times 10^{-11}$ & - \\
\hline 48 & Mannose 1-phosphate & {$[\mathrm{M}-\mathrm{H}]-$} & 259.0226 & 260.0297 & 3.4 & 25.72 & 1.26 & 0.52 & $1.94 \times 10^{-4}$ & - \\
\hline 49 & Dihydroxyacetone phosphate & {$[\mathrm{M}-\mathrm{H}]-$} & 168.9909 & 169.9980 & 5.3 & 26.00 & 1.25 & 0.49 & $3.76 \times 10^{-7}$ & - \\
\hline 50 & Phosphoric acid & {$[\mathrm{M}-\mathrm{H}]-$} & 96.9697 & 97.9769 & 8.2 & 26.94 & 1.88 & 0.52 & $5.17 \times 10^{-10}$ & - \\
\hline 51 & Inosine & {$[\mathrm{M}-\mathrm{H}]-$} & 267.0738 & 268.0808 & 3.7 & 133.96 & 1.57 & 0.08 & $2.83 \times 10^{-11}$ & - \\
\hline 52 & Phenylalanine & {$[\mathrm{M}-\mathrm{H}]-$} & 164.0719 & 165.0789 & 6.1 & 138.26 & 1.51 & 0.51 & $9.47 \times 10^{-7}$ & - \\
\hline 53 & Eicosenoic acid & {$[\mathrm{M}-\mathrm{H}]-$} & 309.2802 & 310.2871 & 3.5 & 553.02 & 1.33 & 0.39 & $1.34 \times 10^{-10}$ & - \\
\hline 54 & 3-Phosphoglyceric acid & {$[\mathrm{M}-\mathrm{H}]-$} & 184.9858 & 185.9929 & 4.9 & 25.46 & 1.44 & 0.08 & $2.54 \times 10^{-8}$ & - \\
\hline 55 & UDP Galactose & {$[\mathrm{M}-\mathrm{H}]-$} & 565.0484 & 566.0550 & 2.5 & 26.29 & 1.63 & 1.59 & $1.99 \times 10^{-3}$ & - \\
\hline 56 & Threonine & {$[\mathrm{M}-\mathrm{H}]-$} & 118.0512 & 119.0582 & 8.4 & 31.39 & 1.02 & 0.50 & $1.09 \times 10^{-6}$ & - \\
\hline 57 & Alanine & {$[\mathrm{M}-\mathrm{H}]-$} & 88.0404 & 89.0476 & 9.0 & 44.24 & 1.28 & 8.18 & $1.22 \times 10^{-7}$ & - \\
\hline 58 & Norleucine & {$[\mathrm{M}-\mathrm{H}]-$} & 130.0875 & 131.0946 & 6.9 & 56.43 & 1.59 & 0.47 & $5.26 \times 10^{-7}$ & - \\
\hline 59 & Uridine 5'-diphosphate & {$[\mathrm{M}-\mathrm{H}]-$} & 402.9955 & 404.0021 & 3.4 & 33.92 & 1.54 & 1.88 & $3.37 \times 10^{-5}$ & - \\
\hline 60 & Pyruvic acid & {$[\mathrm{M}-\mathrm{H}]-$} & 87.0087 & 88.0160 & 7.9 & 30.59 & 1.92 & 0.63 & $2.29 \times 10^{-2}$ & - \\
\hline 61 & Orotidine & {$[\mathrm{M}-\mathrm{H}]-$} & 287.0524 & 288.0594 & 3.5 & 30.02 & 1.75 & 0.67 & $2.52 \times 10^{-3}$ & - \\
\hline 62 & CMP N-acetylneuraminic acid & {$[\mathrm{M}-\mathrm{H}]-$} & 613.1405 & 614.1473 & 1.9 & 26.01 & 2.03 & 0.53 & $2.07 \times 10^{-5}$ & - \\
\hline 63 & UDP glucuronic acid & {$[\mathrm{M}-\mathrm{H}]-$} & 579.0277 & 580.0343 & 2.4 & 23.59 & 2.64 & 2.89 & $1.55 \times 10^{-5}$ & - \\
\hline 64 & N-Acetylneuraminic acid & {$[\mathrm{M}-\mathrm{H}]-$} & 308.0990 & 309.1059 & 3.6 & 28.03 & 1.98 & 1.75 & $5.51 \times 10^{-6}$ & - \\
\hline 65 & UDP-N-acetylglucosamine & {$[\mathrm{M}-\mathrm{H}]-$} & 606.0751 & 607.0815 & 2.6 & 27.07 & 3.06 & 6.12 & $1.28 \times 10^{-8}$ & - \\
\hline 66 & Cholesterol sulfate & {$[\mathrm{M}-\mathrm{H}]-$} & 465.3048 & 466.3116 & 2.6 & 446.27 & 1.57 & 1.27 & $7.72 \times 10^{-5}$ & - \\
\hline 67 & Fumaric acid & {$[\mathrm{M}-\mathrm{H}]-$} & 115.0038 & 116.0109 & 7.8 & 25.15 & 2.26 & 0.54 & $1.84 \times 10^{-7}$ & - \\
\hline 68 & Gluconic acid & {$[\mathrm{M}-\mathrm{H}]-$} & 195.0512 & 196.0583 & 4.6 & 27.64 & 2.49 & 0.34 & $4.36 \times 10^{-5}$ & - \\
\hline
\end{tabular}

a. RT, retention time; b. VIP, variable importance in projection; c. FC, fold change (NCI N87R/ $\triangle$ GATA6 vs. NCI N87R); d. ESI, electrospray ionization.

\section{Metabolic pathways analysis}

To better understand the underlying mechanisms of trastuzumab resistance attenuated by GATA6 knockout in gastric cancer cells, all differential metabolites in NCI N87R/ $\triangle$ GATA6 and MKN45R/ $\triangle$ GATA6 cells were imported into
MetaboAnalyst (version 4.0) for metabolic analysis, respectively. Accordingly, the pathway library of Homo sapiens and Fisher's exact test were applied for pathway enrichment analysis, and relativebetweeness centrality was performed for pathway topology analysis based on reported protocols [17]. The pathway impact values were calculated using 
cumulative percentage according to matched metabolites, and $p$ values were acquired by enrichment analysis based on false FDR. The influenced metabolic pathways were set as pathway impact values more than 0.10 and $p$ value less than 0.05 . According to the $p$ and pathway impact values, ten metabolic pathways were observed in NCI N87R/ $\triangle$ GATA6 cells (Fig. 4A, Table 3). Among them, three pathways were involved in carbohydrate metabolism: (I) citrate cycle, (II) amino sugar and nucleotide sugar metabolism and (III) glycolysis. Four pathways were related to amino acid metabolism: (I) glutamine and glutamate, (II) arginine biosynthesis, (III) arginine and proline and (IV) alanine, aspartate and glutamate metabolism. Moreover, one pathway was subjected to lipid metabolism which involved glycerophospholipid metabolism. We also found that purine metabolism was particularly altered but possessed a low impact value $(p=0.004$, pathway impact value $=0.101)$. Notably, among these pathways, alanine, aspartate and glutamate metabolism displayed the lowest $p$ value $\left(p=9.71 \times 10^{-5}\right)$, whereas glutamine and glutamate metabolism showed highest pathway impact value (pathway impact value $=0.50$ ). Correspondingly, seven pathways showed prominent changes in MKN45R/DGATA6 cells compared to MKN45R cells (Fig. 4B, Table 4). Out of which, three metabolic pathways were relevant to carbohydrate metabolism pathways: (I) glycolysis, (II) amino sugar and nucleotide sugar metabolism, and (III) TCA cycle. Two pathways were part of amino acid metabolism: (I) arginine biosynthesis, and (II) alanine, aspartate and glutamate metabolism. Similarly, glycerophospholipid metabolism was also observed in MKN45R/ $\triangle$ GATA6 cells. In contrast, rather than purine metabolism, pyrimidine metabolism showed a significant change solely in MKN45R/ $\triangle$ GATA6 cells. Apparently, as it can be interpreted from the Venn diagram, six disturbed metabolic pathways were shared by both NCI N87R/ $\triangle$ GATA6 and MKN45R/ $\triangle$ GATA6 cells, accounting for $54.54 \%$ of all pathways altered in these two groups. These pathways include (I) alanine, aspartate and glutamate metabolism, (II) amino sugar and nucleotide sugar metabolism, (III) arginine biosynthesis, (IV) glycerophospholipid metabolism, (V) glycolysis, and (VI) TCA cycle (Fig. 4C). Conversely, some pathways exhibited distinct characteristics as a result of pyrimidine metabolism which was noticeably changed in MKN45R/DGATA6 cells. However, some pathways such as glyoxylate and dicarboxylate metabolism along with arginine and proline metabolism occurred exclusively in NCI N87R/ $\triangle$ GATA6 cells (Fig. 4C). Next, KEGG database (version 89.1) was employed to portray integrated metabolic pathway map to reveal the most relevant metabolic pathways and their potential functions in GATA6 knock out groups based on pathway enrichment and topology analysis. The results were presented manually by drawing united metabolic pathway networks (Figs. 5 \& 6). Noticeably, all metabolic pathways were strongly associated with those related to energy metabolism such as TCA cycle, amino acid metabolism, glycolysis and nucleotide metabolism, indicating that GATA6 modulate the energy metabolism by regulating various metabolic pathways. Collectively, these disturbed metabolic pathways have afforded a deep insight of the mechanisms involved in trastuzumab resistance of gastric cancer cells.

Table 2. Significantly changed metabolites in MKN45R/ $\triangle$ GATA6 compared with MKN45R cells

\begin{tabular}{|c|c|c|c|c|c|c|c|c|c|c|}
\hline No. & Name & Adduct & Observed mass & Exact mass & Mass error (ppm) & $\mathrm{RT}(\mathrm{s})^{\mathrm{a}}$ & VIP value ${ }^{b}$ & $\mathrm{FC}^{\mathrm{c}}$ & $p$ value & ESI ${ }^{\mathrm{d}}$ \\
\hline 1 & 5'-methylthioadenosine & {$[\mathrm{M}+\mathrm{H}]+$} & 298.0963 & 297.0895 & -4.0 & 35.84 & 2.25 & 0.70 & $3.73 \times 10^{-5}$ & + \\
\hline 2 & Adenine & {$[\mathrm{M}+\mathrm{H}]+$} & 136.0617 & 135.0545 & -5.9 & 44.44 & 15.42 & 0.45 & $1.73 \times 10^{-8}$ & + \\
\hline 3 & Creatine & {$[\mathrm{M}+\mathrm{H}]+$} & 132.0767 & 131.0695 & -6.1 & 35.70 & 2.78 & 0.73 & $2.81 \times 10^{-5}$ & + \\
\hline 4 & Guanosine & {$[\mathrm{M}+\mathrm{H}]+$} & 284.0984 & 283.0917 & -4.6 & 135.82 & 7.96 & 0.16 & $2.38 \times 10^{-12}$ & + \\
\hline 5 & Niacinamide & {$[\mathrm{M}+\mathrm{H}]+$} & 123.0552 & 122.0480 & -6.6 & 59.26 & 3.15 & 0.62 & $7.93 \times 10^{-5}$ & + \\
\hline 6 & Pyroglutamic acid & {$[\mathrm{M}+\mathrm{H}]+$} & 130.0498 & 129.0425 & -5.4 & 31.94 & 3.84 & 1.76 & $5.57 \times 10^{-12}$ & + \\
\hline 7 & Histidine & {$[\mathrm{M}+\mathrm{H}]+$} & 156.0767 & 155.0695 & -5.2 & 30.31 & 1.79 & 1.52 & $1.91 \times 10^{-9}$ & + \\
\hline 8 & Citicoline & {$[\mathrm{M}+\mathrm{H}]+$} & 489.1135 & 488.1073 & -3.7 & 35.33 & 1.36 & 0.62 & $2.02 \times 10^{-6}$ & + \\
\hline 9 & Nicotinamide & {$[\mathrm{M}+\mathrm{H}]+$} & 123.0552 & 122.0480 & -6.6 & 40.88 & 2.39 & 0.76 & 0.02 & + \\
\hline 10 & Acetylcarnitine & {$[\mathrm{M}+\mathrm{H}]+$} & 204.1229 & 203.1157 & -3.9 & 56.95 & 2.78 & 0.61 & $2.79 \times 10^{-5}$ & + \\
\hline 11 & Phosphorylcholine & {$[\mathrm{M}]+$} & 184.0732 & 184.0738 & -3.3 & 56.18 & 1.86 & 0.50 & $1.09 \times 10^{-7}$ & + \\
\hline 12 & Glycerophosphocholine & {$[\mathrm{M}+\mathrm{H}]+$} & 258.1098 & 257.1028 & -3.9 & 31.76 & 3.14 & 0.76 & $1.74 \mathrm{E}^{-5}$ & + \\
\hline 13 & Palmitoylethanolamide & {$[\mathrm{M}+\mathrm{H}]+$} & 300.2892 & 299.2824 & -4.0 & 480.69 & 1.67 & 1.71 & $3.47 \times 10^{-9}$ & + \\
\hline 14 & Carnitine & {$[\mathrm{M}+\mathrm{H}]+$} & 162.1123 & 161.1052 & -5.6 & 34.32 & 1.75 & 0.58 & $2.22 \times 10^{-8}$ & + \\
\hline 15 & S-Adenosylmethionine & {$[\mathrm{M}]+$} & 399.1437 & 399.1451 & -3.5 & 36.27 & 1.70 & 0.58 & $4.07 \times 10^{-7}$ & + \\
\hline 16 & Tyrosine & {$[\mathrm{M}+\mathrm{H}]+$} & 182.0811 & 181.0739 & -4.4 & 70.53 & 1.08 & 1.28 & 0.002 & + \\
\hline 17 & Propionylcarnitine & {$[\mathrm{M}+\mathrm{H}]+$} & 218.1384 & 217.1314 & -4.6 & 133.77 & 2.23 & 0.62 & $1.62 \times 10^{-6}$ & + \\
\hline 18 & Cysteinylglycine & {$[\mathrm{M}+\mathrm{H}]+$} & 179.0484 & 178.0412 & -4.5 & 44.90 & 5.83 & 0.59 & $1.99 \times 10^{-7}$ & + \\
\hline 19 & 2-Methylbutyroylcarnitine & {$[\mathrm{M}+\mathrm{H}]+$} & 246.1697 & 245.1627 & -4.1 & 196.15 & 1.46 & 0.46 & $1.20 \times 10^{-8}$ & + \\
\hline 20 & Guanine & {$[\mathrm{M}+\mathrm{H}]+$} & 152.0566 & 151.0494 & -5.3 & 135.83 & 2.94 & 0.18 & $2.53 \times 10^{-13}$ & + \\
\hline
\end{tabular}




\begin{tabular}{|c|c|c|c|c|c|c|c|c|c|c|}
\hline No. & Name & Adduct & Observed mass & Exact mass & Mass error (ppm) & RT $(\mathrm{s})^{\mathrm{a}}$ & VIP value ${ }^{b}$ & FCc & $p$ value & ESI $^{d}$ \\
\hline 21 & Lauryl diethanolamide & {$[\mathrm{M}+\mathrm{H}]+$} & 288.2529 & 287.2460 & -3.8 & 379.18 & 2.05 & 1.43 & $3.37 \times 10^{-5}$ & + \\
\hline 22 & Tetradecanoylcarnitine & {$[\mathrm{M}+\mathrm{H}]+$} & 372.31 & 371.3036 & -4.3 & 541.29 & 1.07 & 0.07 & $5.93 \times 10^{-11}$ & + \\
\hline 23 & Glutamine & {$[\mathrm{M}+\mathrm{H}]+$} & 147.0763 & 146.0691 & -5.5 & 31.566 & 7.06 & 0.08 & $8.91 \times 10^{-15}$ & + \\
\hline 24 & PC (14:0/16:0) & {$[\mathrm{M}+\mathrm{H}]+$} & 706.5376 & 705.5308 & -1.7 & 380.78 & 1.72 & 0.71 & $5.03 \times 10^{-3}$ & + \\
\hline 25 & Adenosine & {$[\mathrm{M}+\mathrm{H}]+$} & 268.1036 & 267.0968 & -4.5 & 135.79 & 2.32 & 0.09 & $9.63 \times 10^{-12}$ & + \\
\hline 26 & Sphinganine & {$[\mathrm{M}+\mathrm{H}]+$} & 302.3047 & 301.2980 & -4.3 & 534.68 & 2.06 & 0.72 & $1.02 \times 10^{-2}$ & + \\
\hline 27 & LysoPE (16:0/0:0) & {$[\mathrm{M}+\mathrm{H}]+$} & 454.2919 & 453.2855 & -3.5 & 416.32 & 1.35 & 1.53 & $2.87 \times 10^{-7}$ & + \\
\hline 28 & LysoPE (18:1(11Z)/0:0) & {$[\mathrm{M}+\mathrm{H}]+$} & 480.3076 & 479.3012 & -3.3 & 428.31 & 1.16 & 1.28 & $3.1 \times 10^{-2}$ & + \\
\hline 29 & Citric/Isocitric acid & {$[\mathrm{M}-\mathrm{H}]-$} & 191.0199 & 192.0270 & 4.7 & 27.65 & 2.58 & 0.68 & $2.04 \times 10^{-5}$ & - \\
\hline 30 & Xanthine & {$[\mathrm{M}-\mathrm{H}]-$} & 151.0263 & 152.0334 & 5.9 & 58.40 & 4.10 & 0.52 & $8.04 \times 10^{-3}$ & - \\
\hline 31 & Phosphoenolpyruvic acid & {$[\mathrm{M}-\mathrm{H}]-$} & 166.9752 & 167.9823 & 5.4 & 24.84 & 1.50 & 0.23 & $6.66 \times 10^{-8}$ & - \\
\hline 32 & Malic acid & {$[\mathrm{M}-\mathrm{H}]-$} & 133.0144 & 134.0215 & 6.7 & 25.33 & 3.12 & 0.78 & $4.22 \times 10^{-6}$ & - \\
\hline 33 & Eicosadienoic Acid & {$[\mathrm{M}-\mathrm{H}]-$} & 307.2646 & 308.2715 & 3.6 & 517.33 & 2.52 & 1.59 & 0.02 & - \\
\hline 34 & Glyceric acid & {$[\mathrm{M}-\mathrm{H}]-$} & 105.0194 & 106.0266 & 7.5 & 27.37 & 4.40 & 2.74 & $1.14 \times 10^{-11}$ & - \\
\hline 35 & Mannose 1-phosphate & {$[\mathrm{M}-\mathrm{H}]-$} & 259.0226 & 260.0297 & 3.5 & 25.72 & 9.64 & 1.45 & $2.10 \times 10^{-3}$ & - \\
\hline 36 & Dihydroxyacetone phosphate & {$[\mathrm{M}-\mathrm{H}]-$} & 168.9909 & 169.9980 & 5.3 & 26.00 & 2.31 & 0.57 & $6.37 \times 10^{-5}$ & - \\
\hline 37 & Inosine & {$[\mathrm{M}-\mathrm{H}]-$} & 267.0738 & 268.0808 & 3.7 & 133.96 & 7.21 & 0.45 & $1.03 \times 10^{-3}$ & - \\
\hline 38 & N-Acetyl-L-aspartic acid & {$[\mathrm{M}-\mathrm{H}]-$} & 174.0409 & 175.0481 & 4.6 & 25.90 & 2.26 & 0.69 & $9.48 \times 10^{-9}$ & - \\
\hline 39 & Pantothenic Acid & {$[\mathrm{M}-\mathrm{H}]-$} & 218.1036 & 219.1107 & 4.1 & 44.68 & 2.10 & 0.63 & $7.28 \times 10^{-6}$ & - \\
\hline 40 & Uridine 5'-monophosphate & {$[\mathrm{M}-\mathrm{H}]-$} & 323.0288 & 324.0358 & 3.1 & 29.44 & 9.26 & 1.90 & 0.01 & - \\
\hline 41 & Eicosenoic acid & {$[\mathrm{M}-\mathrm{H}]-$} & 309.2802 & 310.2872 & 3.2 & 553.01 & 1.65 & 1.43 & 0.02 & - \\
\hline 42 & 3-Phosphoglyceric acid & {$[\mathrm{M}-\mathrm{H}]-$} & 184.9858 & 185.9929 & 4.9 & 25.46 & 2.12 & 0.16 & $2.06 \times 10^{-9}$ & - \\
\hline 43 & Glyceraldehyde & {$[\mathrm{M}-\mathrm{H}]-$} & 89.0245 & 90.0316 & 10 & 28.46 & 1.49 & 0.75 & $1.12 \times 10^{-4}$ & - \\
\hline 44 & UDP-galactose & {$[\mathrm{M}-\mathrm{H}]-$} & 565.0484 & 566.0550 & 2.5 & 26.29 & 1.01 & 1.55 & $6.48 \times 10^{-5}$ & - \\
\hline 45 & Threonine & {$[\mathrm{M}-\mathrm{H}]-$} & 118.0512 & 119.0582 & 8.4 & 31.39 & 4.74 & 1.28 & $8.37 \times 10^{-5}$ & - \\
\hline 46 & Eicosapentaenoic Acid & {$[\mathrm{M}-\mathrm{H}]-$} & 301.2176 & 302.2245 & 3.6 & 432.09 & 4.45 & 1.65 & $1.51 \times 10^{-6}$ & - \\
\hline 47 & ADP & {$[\mathrm{M}-\mathrm{H}]-$} & 426.0227 & 427.0294 & 3.0 & 41.64 & 2.07 & 0.57 & $2.37 \times 10^{-5}$ & - \\
\hline 48 & Alanine & {$[\mathrm{M}-\mathrm{H}]-$} & 88.0404 & 89.0476 & 9.0 & 44.24 & 1.88 & 1.42 & $1.49 \times 10^{-5}$ & - \\
\hline 49 & Uridine 5'-diphosphate & {$[\mathrm{M}-\mathrm{H}]-$} & 402.9955 & 404.0022 & 3.2 & 33.92 & 1.74 & 2.13 & $5.0 \times 10^{-4}$ & - \\
\hline 50 & Arachidonic acid & {$[\mathrm{M}-\mathrm{H}]-$} & 303.2333 & 304.2402 & 3.6 & 462.07 & 4.68 & 1.67 & $3.67 \times 10^{-7}$ & - \\
\hline 51 & Orotidine & {$[\mathrm{M}-\mathrm{H}]-$} & 287.0524 & 288.0594 & 3.5 & 30.01 & 5.26 & 0.57 & $1.03 \times 10^{-5}$ & - \\
\hline 52 & CMP N-acetylneuraminic acid & {$[\mathrm{M}-\mathrm{H}]-$} & 613.1405 & 614.1473 & 1.9 & 26.00 & 1.97 & 0.70 & $1.57 \times 10^{-5}$ & - \\
\hline 53 & UDP glucuronic acid & {$[\mathrm{M}-\mathrm{H}]-$} & 579.0277 & 580.0343 & 2.4 & 23.58 & 1.23 & 2.34 & $4.93 \times 10^{-9}$ & - \\
\hline 54 & N-Acetylneuraminic acid & {$[\mathrm{M}-\mathrm{H}]-$} & 308.0990 & 309.1059 & 3.6 & 28.02 & 1.88 & 1.65 & $1.40 \times 10^{-9}$ & - \\
\hline 55 & UDP-N-acetylglucosamine & {$[\mathrm{M}-\mathrm{H}]-$} & 606.0751 & 607.0815 & 2.6 & 27.06 & 3.17 & 1.86 & $8.43 \times 10^{-10}$ & - \\
\hline 56 & Uracil & {$[\mathrm{M}-\mathrm{H}]-$} & 111.0201 & 112.0273 & 7.1 & 31.07 & 6.44 & 0.59 & 0.003 & - \\
\hline 57 & Fumaric acid & {$[\mathrm{M}-\mathrm{H}]-$} & 115.0038 & 116.0109 & 7.8 & 25.14 & 2.15 & 1.24 & $1.74 \times 10^{-6}$ & - \\
\hline 58 & Gluconic acid & {$[\mathrm{M}-\mathrm{H}]-$} & 195.0512 & 196.0583 & 4.6 & 27.64 & 3.73 & 1.29 & 0.001 & - \\
\hline 59 & Citrulline & {$[\mathrm{M}-\mathrm{H}]-$} & 174.0886 & 175.0956 & 5.7 & 31.65 & 1.44 & 0.71 & 0.002 & - \\
\hline
\end{tabular}

a. RT, retention time; b. VIP, variable importance in projection; c. FC, fold change (MKN45R/ $\triangle \mathrm{GATA6} v s . \mathrm{MKN} 45 \mathrm{R})$; d. ESI, electrospray ionization.

Table 3. Ten prominent metabolic pathways enriched via MetaboAnalyst based on differential metabolites identified in $\mathrm{NCl}$ N87R/

$\triangle$ GATA6 vs. $\mathrm{NCl} \mathrm{N87R} \mathrm{cells}$

\begin{tabular}{lllll}
\hline No. & Pathway name & $\begin{array}{l}\text { Hits/ } \\
\text { Total }\end{array}$ & $p$ values & $\begin{array}{l}\text { Pathway } \\
\text { impact values }\end{array}$ \\
\hline 1 & $\begin{array}{l}\text { Alanine, aspartate and glutamate } \\
\text { metabolism }\end{array}$ & $7 / 28$ & $9.71 \times 10^{-5}$ & 0.399 \\
2 & Arginine biosynthesis & $4 / 14$ & $2.06 \times 10^{-3}$ & 0.345 \\
3 & Purine metabolism & $8 / 65$ & $4.65 \times 10^{-3}$ & 0.101 \\
4 & TCA cycle & $4 / 20$ & $8.22 \times 10^{-3}$ & 0.166 \\
5 & Glyoxylate and dicarboxylate metabolism & $5 / 32$ & $9.29 \times 10^{-3}$ & 0.111 \\
6 & Glycerophospholipid metabolism & $5 / 36$ & $1.53 \times 10^{-2}$ & 0.121 \\
7 & Amino sugar and nucleotide sugar & $5 / 37$ & $1.71 \times 10^{-2}$ & 0.139 \\
& metabolism & & & \\
8 & Arginine and proline metabolism & $5 / 38$ & $1.91 \times 10^{-2}$ & 0.176 \\
9 & Glutamine and glutamate metabolism & $2 / 6$ & $2.33 \times 10^{-2}$ & 0.50 \\
10 & Glycolysis & $4 / 26$ & $2.21 \times 10^{-2}$ & 0.206 \\
\hline
\end{tabular}

\section{Integrated analysis of metabolomics and proteomics}

To further unveil the potential metabolism- related mechanisms responsible for GATA6-mediated trastuzumab resistance, we constructed an integrated metabolite-protein regulatory network from differential metabolites and target genes based on the OmicsNet (version 1.0) [18], from which the results were annotated using a visual network diagram (Fig. 7). We observed that fifteen crucial differential metabolites were closely related to TCA cycle, energy metabolism and nucleotide metabolism, including phosphoenolpyruvate, citrate/isocitrate, fumarate, pyruvate, glutamate, glutamine, 3-phosphoglyceric acid (3-PG), glycerate, adenine, guanine, xanthine, GMP, guanosine, adenosine and inosine. Phosphoenolpyruvate, which is involved in glycolysis and gluconeogenesis, exhibited a dramatic decrease in both NCI N87R/DGATA6 and MKN45R/DGATA6 cells. Furthermore, integrated data showed that phosphoenolpyruvate was regulated by nine molecules including PCK2, ENO1, NANS, ENO3, PKM, PKLR, ENO4, PCK1 and ENO2. Among them, PCK2 and 
NANS were increased, whereas conversely, ENO1, ENO3, PKM and PKLR were decreased in NCI N87R/ $\triangle$ GATA6 cells. Similarly, thirty target proteins regulate pyruvate, another key intermediate metabolite in carbohydrate, protein and fat metabolism that regulates sugar metabolism out of which PKLR, PKM, DLAT, LDHA and ME2 were downregulated while PDHB, PDHA1 were upregulated in NCI N87R/ $\triangle$ GATA6 cells. We also noticed that RKLR and PKM were co-regulatory molecules in pyruvate-phosphoenolpyruvate subnetwork. Similarly, fumarate, a precursor to L-malate in TCA cycle, which is converted by the enzyme fumarase to malate, was regulated by SDHB, SDHA, FAH, ADSL, FH, SDHAF2, ASL and FAHD1. From these molecules only ASL displayed an obvious upregulation, whereas, SDHAF2 showed a distinct down-regulation in NCI N87R/ $\Delta$ GATA6 cells. Additionally, we observed that five different molecules regulated citrate with only ACO2 showing significant down-regulation whereas no significant changes occurred in the expression of CS, ACO1, ACLY and RIMKLB in NCI N87R/ $\triangle$ GATA6 cells, indicating that $\mathrm{ACO} 2$ plays a major role in regulating citrate. Moreover, we noticed that 3-PG, which is a significant metabolic intermediate in both glycolysis and TCA cycle, reduced sharply in GATA6 knock out cells. It was regulated by some typical molecules including ACP1, PDP1, GNPAT, ALDOA, ALDOC, ALDH2, ALDH1B1 and ME2, which were all altered significantly. Meanwhile, we found eight different molecules modulated glycerate, out of which ALDH2, ALDH1B1 and ME2 showed prominent downregulation in NCI N87R/ $\triangle$ GATA6 cells. According to the sub-network, we also observed that ME2 is a co-regulatory molecule of pyruvate, 3-PG and glycerate. Besides, when compared with NCI N87R cells, our data revealed some factors that regulate glutamate and glutamine. GLUD1, GGH, ALDH18A1, ALDH4A1, GMPS and CAD displayed a prominent up regulation, in contrast, GCLC, GOT1, GOT2, PSAT1, CTPS2, GFPT1, GLS and GLUL showed dramatic down regulation in NCI N87R/ $\triangle$ GATA6 cells. Several molecules, which regulate adenine, guanine, xanthine, GMP, guanosine, adenosine and inosine, were also changed dramatically in which AHCYL1 and DCK were decreased, while MTAP, GDA, HPRT1, APRT, PNP and ADA were increased instead (Fig. 7). Next, we employed the STRING database (version 11.0) to construct interactive networks of activated transcription factors in NCI N87R/AGATA6 cells, whose interactions have high confidence with scores more than 0.70 [19]. We noticed that PKM and PKLR represented primary hubs in regulating TCA cycle, glycolysis and energy metabolism, GLUL and GLS displayed another center in regulating amino acid metabolism, and APRT represented a main hub in regulating nucleotide metabolism (Fig. 8A). Quantification of these transcription factors using proteomics are shown in Fig. 8B. Thereafter, we confirmed the expression alteration of PKM, GLS1, GLUL and LDHA in NCI

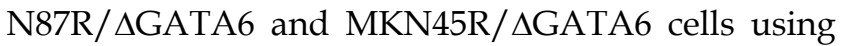
western blotting, and consistent with our mass spectrometry data, a remarkable down regulation of PKM, GLUL, GLS1 and LDHA were observed in GATA6 knock out groups as compared to their resistant groups (Fig. 8C, D).

Table 4. Seven prominent metabolic pathways enriched via MetaboAnalyst based on differential metabolites identified in MKN45R/AGATA6 vs. MKN45R cells

\begin{tabular}{|c|c|c|c|c|}
\hline No. & Pathway name & Hits/Total & $p$ values & $\begin{array}{l}\text { Pathway } \\
\text { impact values }\end{array}$ \\
\hline 1 & $\begin{array}{l}\text { Alanine, aspartate and glutamate } \\
\text { metabolism }\end{array}$ & $4 / 28$ & $1.27 \times 10^{-2}$ & 0.203 \\
\hline 2 & Arginine biosynthesis & $3 / 14$ & $9.98 \times 10^{-3}$ & 0.228 \\
\hline 3 & Pyrimidine metabolism & $5 / 65$ & $8.47 \times 10^{-3}$ & 0.270 \\
\hline 4 & TCA cycle & $3 / 20$ & $2.43 \times 10^{-2}$ & 0.129 \\
\hline 5 & Glycerophospholipid metabolism & $5 / 36$ & $5.98 \times 10^{-3}$ & 0.190 \\
\hline 6 & $\begin{array}{l}\text { Amino sugar and nucleotide sugar } \\
\text { metabolism }\end{array}$ & $5 / 37$ & $6.75 \times 10^{-3}$ & 0.139 \\
\hline 7 & Glycolysis & $3 / 26$ & $3.16 \times 10^{-2}$ & 0.106 \\
\hline
\end{tabular}

\section{Discussion}

TCA cycle, considered as the central hub for supervision of energy metabolism for cancer cells, contributes to constant supply of energy for synthesis of proteins, lipid and nucleic acids. Abnormal TCA cycle is involved in several biological processes of cancer cells including aberrant metabolism [20]. Our results showed that TCA cycle was suppressed and led to abnormal energy metabolism as evidenced by a distinct decrease in fumarate, malate and citrate/isocitrate in GATA6 knock out cells (Figs. S5, S6). It is worth noting that relatively lower levels of ATP and MMP were observed in GATA6 knockout groups compared to trastuzumab resistant groups (Fig. 1C, D). Furthermore, proteomics data displayed that expression of mitochondrial aconitase (ACO2), which is an important regulatory enzyme of the TCA cycle, was significantly downregulated in NCI N87R/ $\triangle$ GATA6 cells. Similarly, succinate dehydrogenase assembly factor 2 (SDHAF2), which is a component of both the TCA cycle and the mitochondrial electron transport chain, was also decreased in NCI N87R/ $\triangle$ GATA6 cells when compared with NCI N87R cells. It is well-known that NAD-dependent malic enzyme (ME2) plays a key role in the malate/aspartate shuttle across the mitochondrial membrane involving regulation of redox balance, cellular energy and 
biosynthesis of molecules. Various studies have demonstrated that ME2 overexpression is associated with cell migration and invasion, whereas low expression of ME2 leads to a decrease in synthesis efficiency of DNA, thereby ultimately causing cancer cells apoptosis [21,22]. The present study showed that GATA6 knockout resulted in the down-regulation of

A

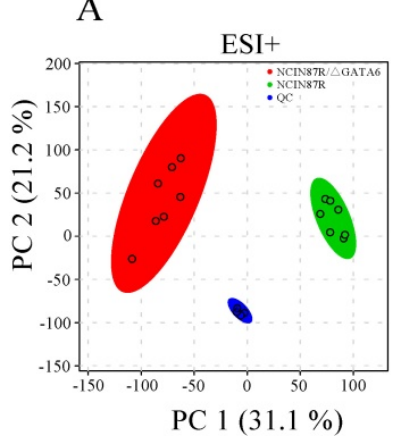

E

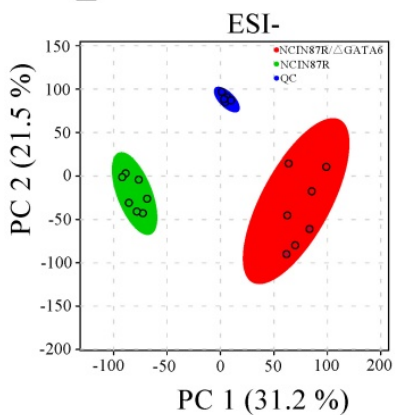

I

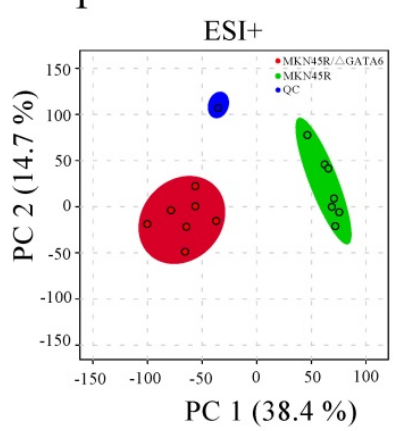

$\mathrm{M}$

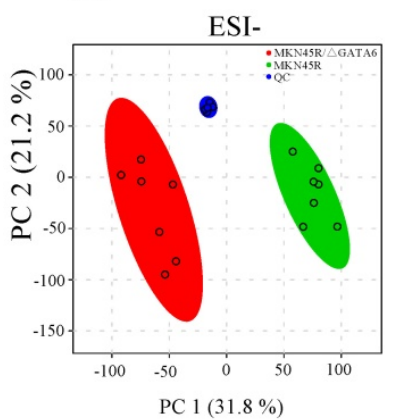

B

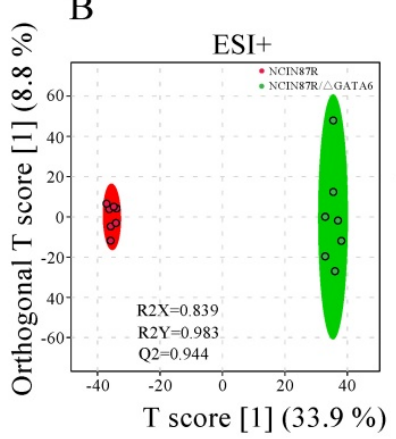

F
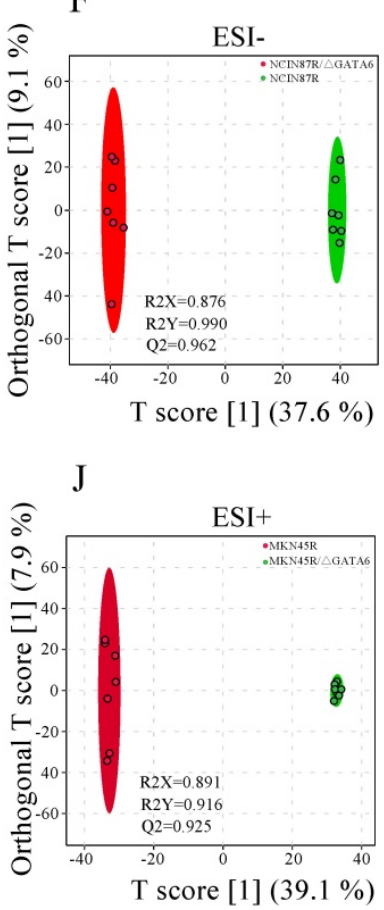

$\mathrm{N}$

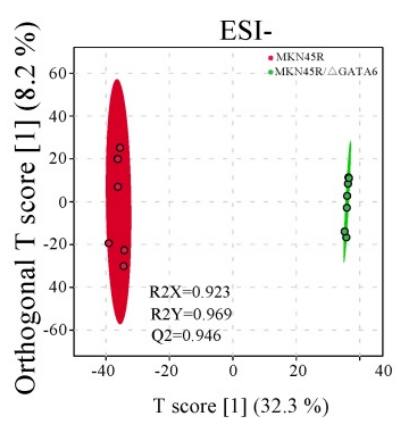

ME2 in trastuzumab resistant gastric cancer cells, which in turn reduced ATP production and ultimately inhibited the proliferation of cells. Taken together, these results suggest that GATA6 contributes to trastuzumab resistance, which is dependent on TCA cycle and mitochondrial function in gastric cancer.

C

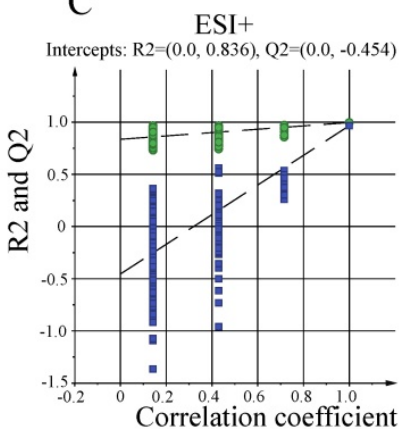

G

ESI-

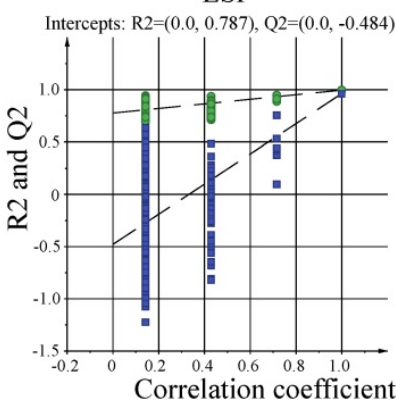

K

ESI+

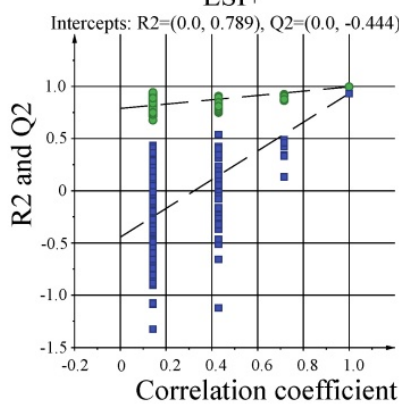

$\mathrm{O}$

$\mathrm{ESI}+$

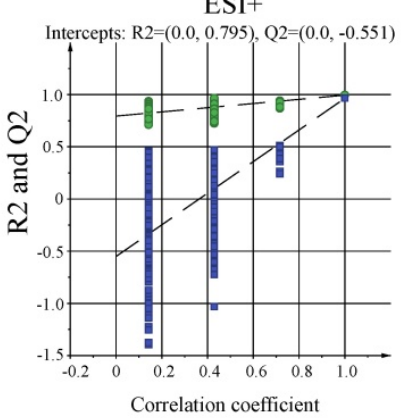

D

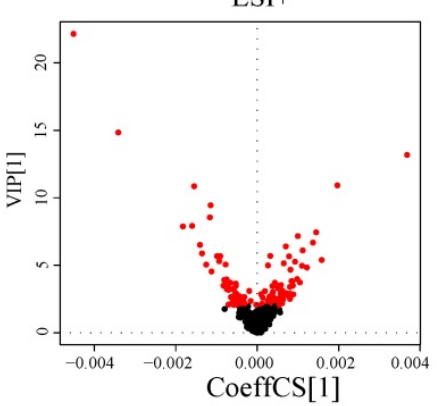

$\mathrm{H}$

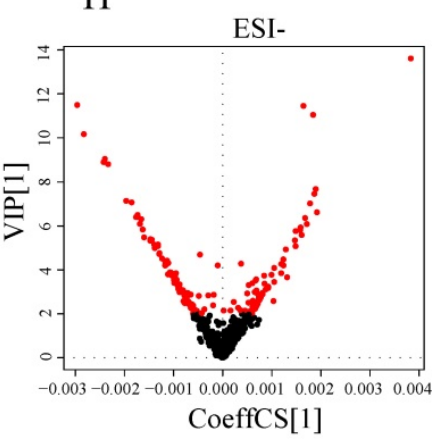

$\mathrm{L}$

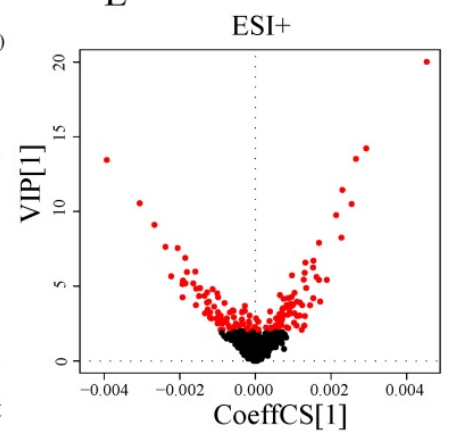

$\mathrm{P}$

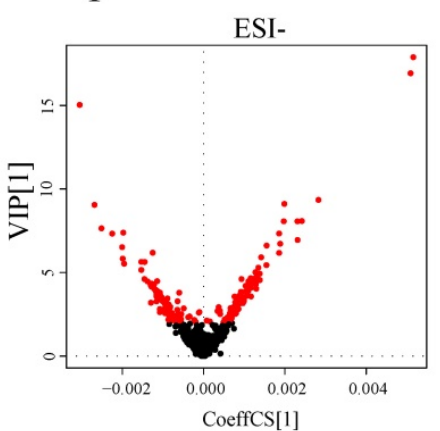

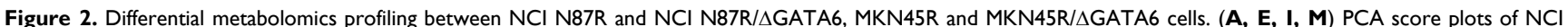

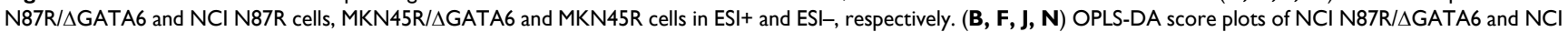
N87R cells, MKN45R/AGATA6 and MKN45R cells in ESI+ and ESI-, respectively. (C, G, K, O) Permutation test plots of NCI N87R/AGATA6 and NCI N87R cells,

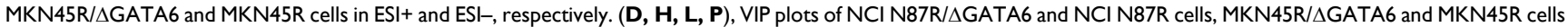
in ESI+ and ESI- respectively, red nodes represent corresponding variables with VIP > 1.0 in each group. 
A

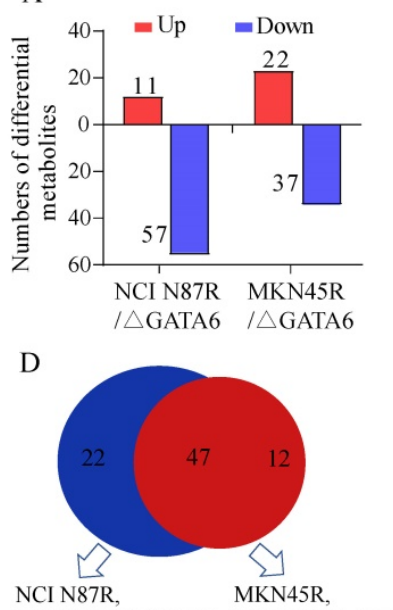

NCI N87R/ $\triangle$ GATA6 MKN45R/ $\triangle$ GATA6

$\mathrm{E}$
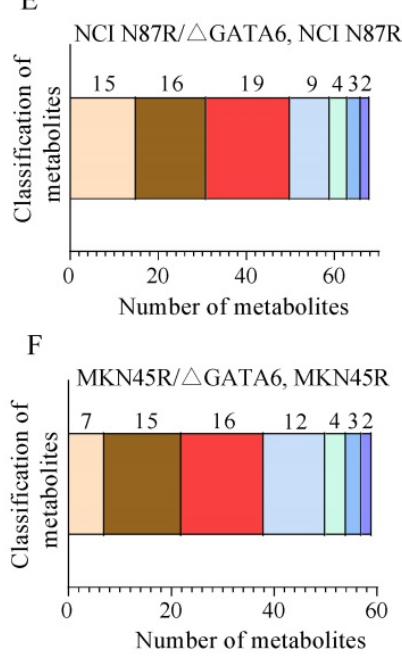

$$
\begin{array}{ll}
\square \text { Others } & \square \text { Amine } \\
\square \text { Carbohydrate } & \square \text { Orgnic acid } \\
\square \text { Cofactor } & \square \text { Nucleotides } \\
\square \text { Amino acid } &
\end{array}
$$

B

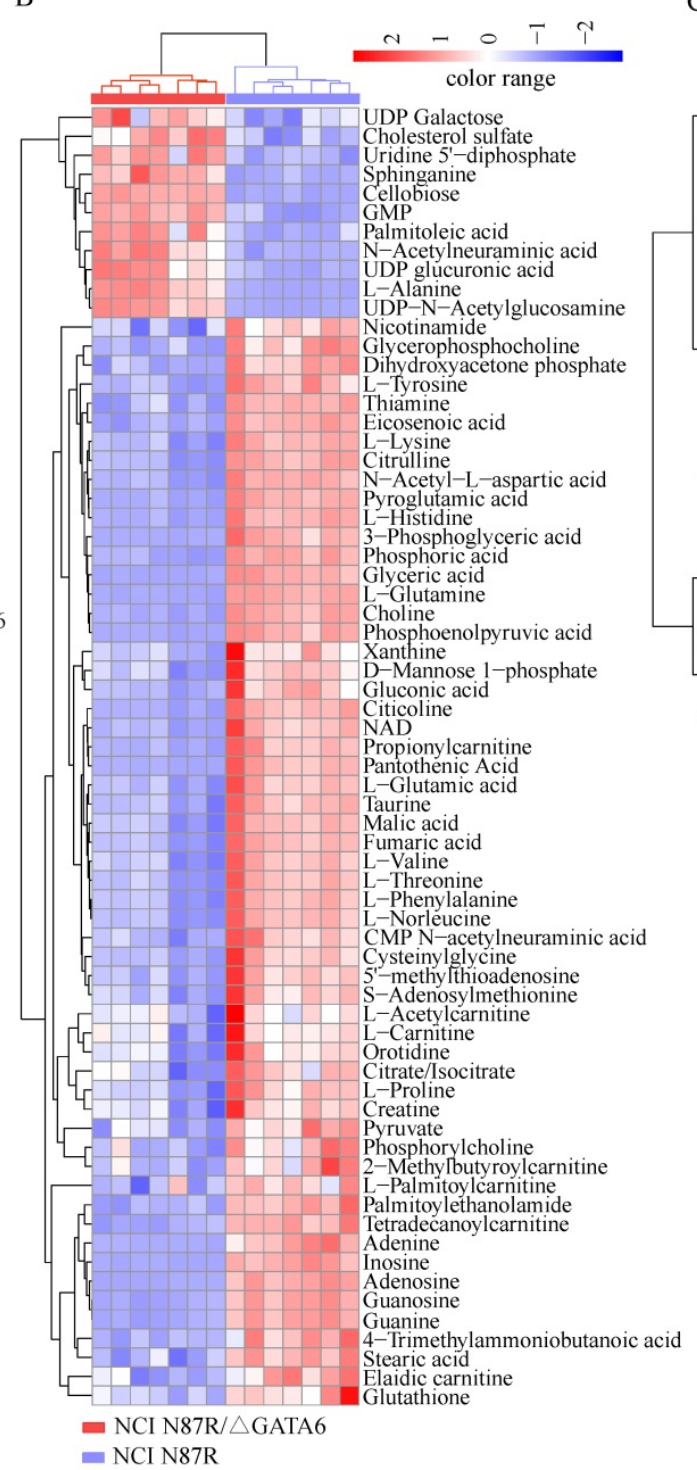

$\mathrm{C}$

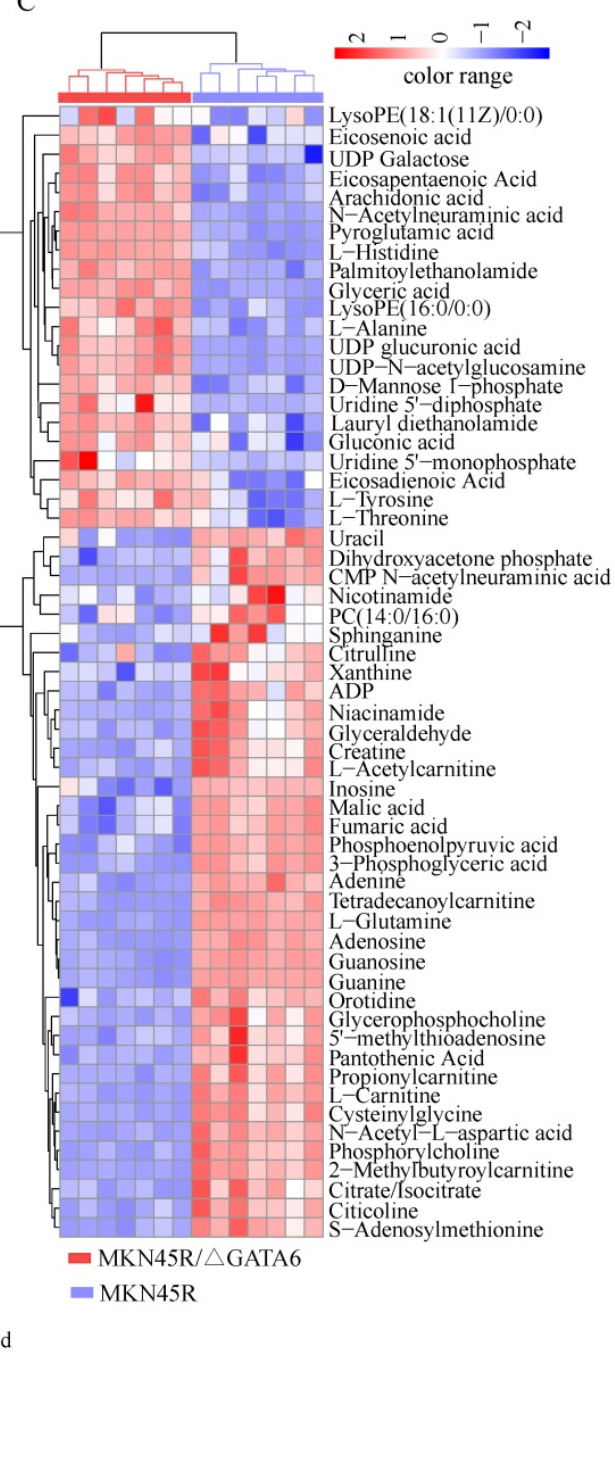

Figure 3. Analysis of differential metabolites between GATA6 knock out groups vs. trastuzumab resistant groups. (A) Increased and decreased metabolites in NCl

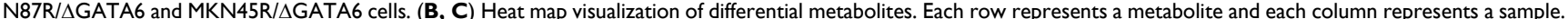
The color scale with red and blue intensity denotes increased and decreased metabolites, respectively. (D) Venn diagram shows the shared and non-shared metabolites among $\mathrm{NCI} N 87 \mathrm{R} / \triangle \mathrm{GATA6}, \mathrm{NCI}$ N87R cells, MKN45R/AGATA6 and MKN45R cells. (E, F) Classification of differential metabolites by their properties.

Cancer cells have higher rate of glycolysis than normal cells in order to generate more ATP for metabolic activities, which is a hallmark of cancer termed as "Warburg effect" and has been widely accepted as a common feature of metabolic reprogramming [23]. Increasing body of evidence has revealed that inhibition of glycolysis in cancer cells is an effective approach to overcome multidrug resistance related to mitochondrial respiratory defect and hypoxia [24]. Multiple molecules involved in glycolysis have been reported to be associated to HER-2 signaling pathway in cancer cells, such as lactate dehydrogenase A (LDHA), a key enzyme in the glycolytic pathway, the level of which is facilitated by overexpression of HER-2 which further enhances the utilization of glucose and decreases the consumption of oxygen in breast cancer cells [25]. Accordingly, inhibition of LDHA activity compromised the tumorigenesis and proliferation of HER-2 initiated cancer cells [26]. Moreover, the high level of glutamine synthetase (GLUL) was positively correlated with the expression of HER-2 and proliferation of cancer cell [27]. Of note, reduced glucose uptake and hexokinase activity were also observed in HER-2 positive breast cancer cells following treatment with trastuzumab [28]. However, present studies are mainly focused on the interplay between HER-2 and glycolysis in the initiation and progression of breast cancers. Hence, the role of HER-2 signaling in the aberrant glucose metabolism of gastric cancer remains to be elucidated. 
A

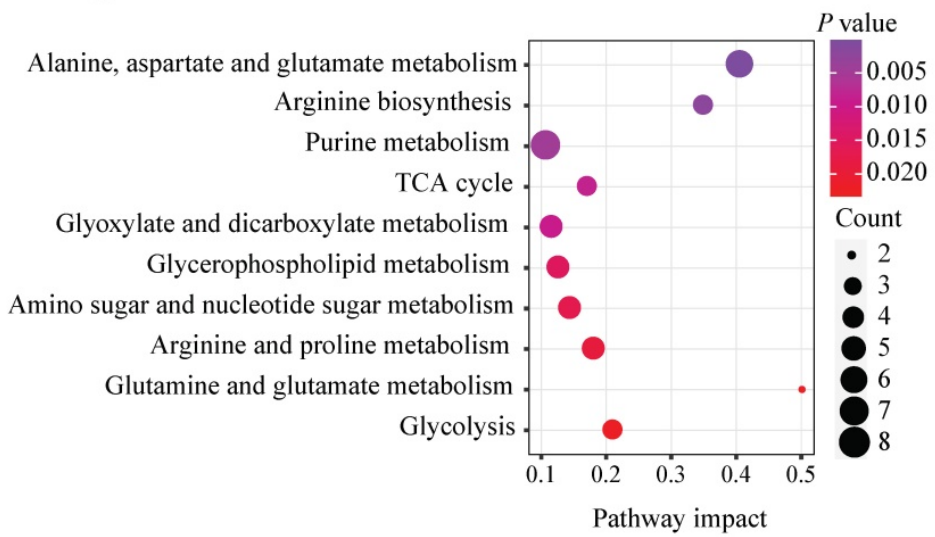

B

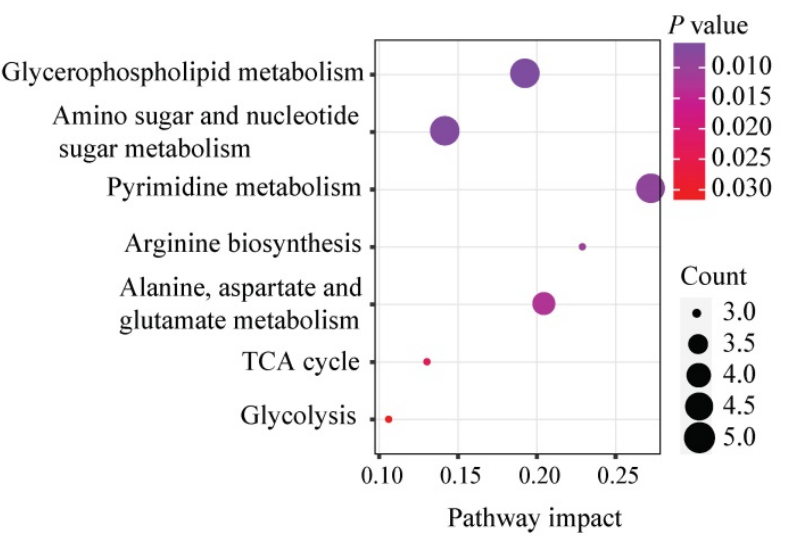

C

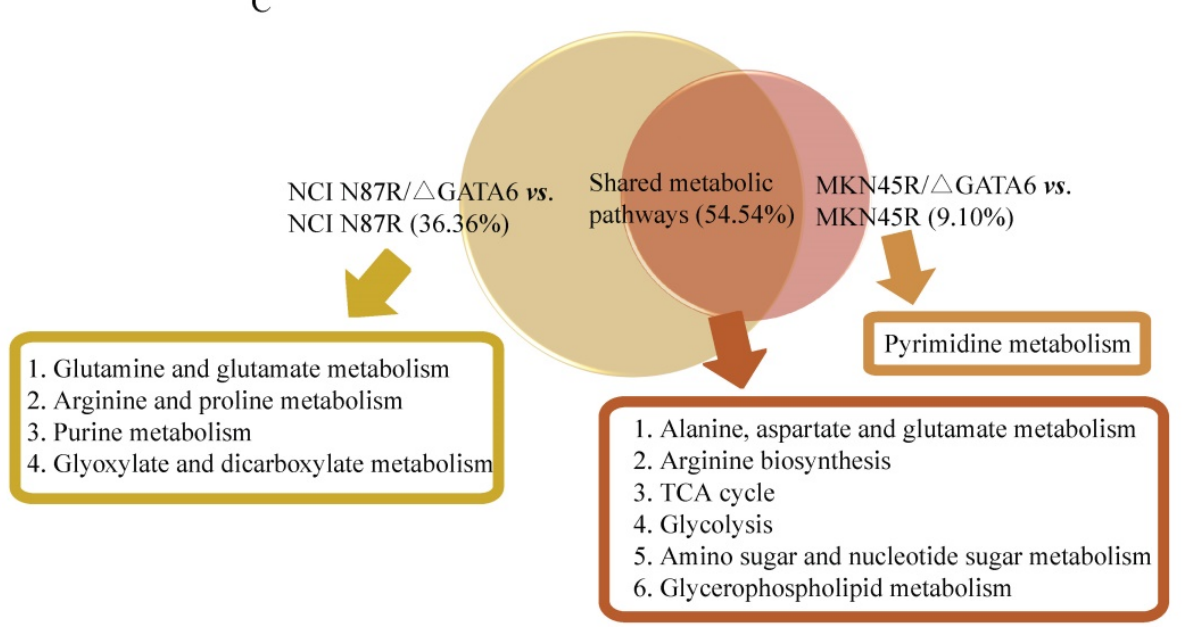

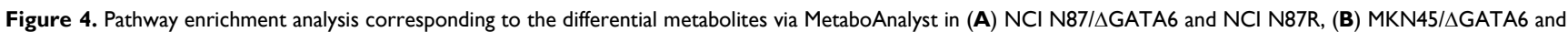
MKN45R cells. $x$-axis represents the pathway impact value of topological analysis. Size of the nodes shows the number of matched metabolites, color of the nodes indicates $p$ value of the enrichment analysis.

In this study, we found that some key metabolites related to glycolysis showed a dramatic decrease in GATA6 knockout groups, including dihydroxyacetone (DHAP), G3P, phosphoenolpyruvate (PEP), and pyruvic acid (Figs. S5, S6), suggesting that GATA6 is one of the key factors in the regulation of glycolysis in trastuzumab resistant gastric cancer cells. Pyruvate kinase $\mathrm{M}$ (PKM) is known to be an important rate-limiting enzyme of the glycolytic pathway, which catalyzes the conversion of PEP and adenosine diphosphate (ADP) into pyruvate and ATP. A previous study has suggested that low level of GATA6 triggers glycolysis by activating PKM in hepatocellular carcinoma [29]. However, unlike the above study, our data showed that GATA6 knockout caused down-regulation of PKM and inhibition of glycolysis. This discrimination might be due to the different cell type (hepatocellular carcinoma vs. gastric cancer). However, other molecular mechanisms should also be taken into account. Knockdown of LDHA in tumor cells induces increased mitochondrial respiration, decreased proliferation and suppressed tumorigenicity [30]. Previous study showed that LDHA has a relatively higher expression in paclitaxel-resistant than paclitaxel- sensitive breast cancer cells, and down-regulation of LDHA can re-sensitizes resistant cells to paclitaxel again [31]. In consistency with this study, we also found that GATA6 knockout led to decrease of LDHA, and subsequently glycolysis was further inhibited in NCI N87R/ $\triangle$ GATA6 and MKN45R/DGATA6 cells. Collectively, it might suggest that GATA6 confers glycolysis by regulating glycolytic related kinase activity and expression. Moreover, we found that amino sugar and nucleotide sugar metabolism was promoted due to increased uridine 5'-diphosphate-galactose (UDP-galactose), uridine diphosphate-N-acetylglucosamine (UDPGlcNAc), N-acetylneuraminic acid (UDP-Neu5Ac), uridine diphosphate (UDP) and UDP-glucuronate levels in the cells with GATA6 knock out. We propose that this might be an alternative mechanism of GATA6 knock out cells, primarily activating the 
amino sugar and nucleotide sugar metabolism to compensate for the inhibited glycolytic pathway for acquiring necessary energy and nutrients.

Mounting evidence has shed light on the important roles of amino acids in metabolism of cancer cells [32]. It is known that glutamate is a nitrogen donor for synthesizing some nitrogenous compounds in cancer cells. Glutamine is a major amino acid that drives the TCA cycle to sustain mitochondrial ATP production in cancer cells [33].
The utilization of glutamine decreased drastically when the cells were under hypoxia or with mitochondrial dysfunction [34]. Proline is a source of carbon exchange between TCA cycle and urea cycle. Arginine serves as the precursor to proline or as an additional source of glutamate. Our results demonstrated lower levels of glutamine, glutamate, $\mathrm{N}$-acetyl-aspartate, citrulline and proline while higher levels of alanine, tyrosine, and phenylalanine in trastuzumab resistant cells with GATA6 knockout

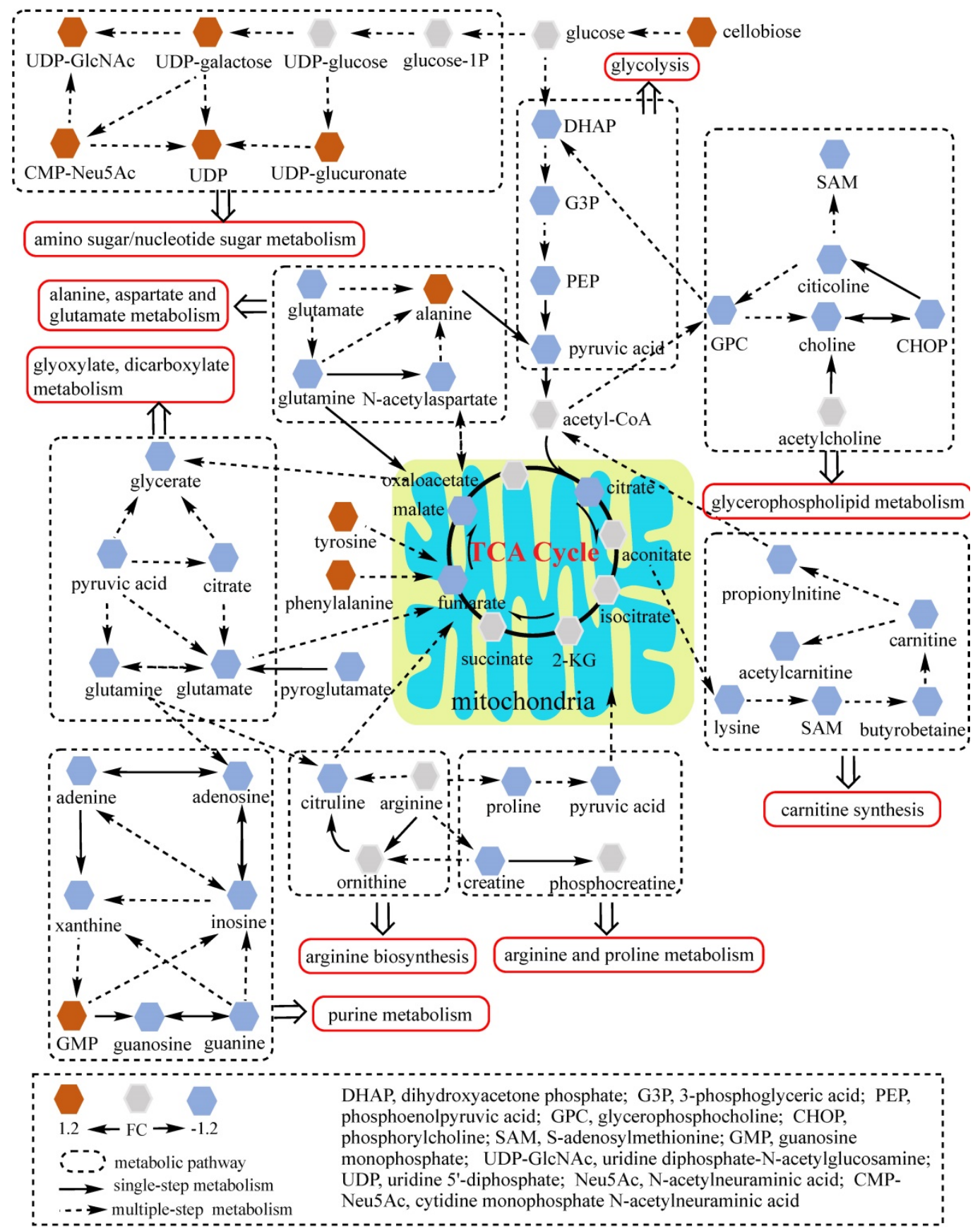

Figure 5. Network analysis of significantly changed metabolic pathways. Ten pathways disturbed in NCI N87R/ $\triangle \mathrm{GATA6}$ cells were analyzed. Each hexagon represents a metabolite, brown and blue ones represent 1.2 fold increase or decrease in NCI N87R/AGATA6 cells respectively. Dashed box indicates different metabolic pathways. Solid arrows show single-step metabolism, and dotted arrows show multiple-step metabolism. 
compared with trastuzumab resistant gastric cancer cells, suggesting that GATA6 plays an important role in amino acid metabolisms of trastuzumab resistant gastric cancer cells. Of note, these altered metabolites belong to glutamate and glutamine metabolism, alanine, aspartate and glutamate metabolism, arginine and proline metabolism, and arginine biosynthesis (Tables $3 \& 4$ ). Furthermore, we analyzed two critical enzymes regulating glutamine and glutamate. Aspartate aminotransferase (GOT1/2), important regulators of levels of glutamate which contributes to glucose synthesis and gluconeogenesis, have been found to be upregulated in various tumor cells [35]. Moreover, in colorectal cancer cell, inhibition of GOT1 activity increases the sensitivity to 5-fluorouracil [36]. Our study showed that GOT1 and GOT2 were decreased in trastuzumab resistant gastric cancer cells with GATA6 knockout, suggesting that GATA6 maintains basal expression of GOT1 and
GOT2. Glutaminase (GLS), which catalyzes the transformation of glutamine to glutamate, is regarded as another critical enzyme in growth and proliferation of cancer cells. A prior study has shown that silencing of GLS leads to re-sensitization of taxol-resistant breast cancer, suggesting its key role in taxolresistance [37]. Consistently, we found that GATA6 knockout resulted in decreased expression of GLS and thereupon disturbed glutamate and glutamine metabolism in trastuzumab resistant gastric cancer cells. Furthermore, several amino acid metabolic pathways were altered in NCI N87R/ $\Delta$ GATA6 cells including arginine and proline metabolism and arginine biosynthesis. Collectively, we speculated that GATA6 regulates multiple amino acid metabolism pathways, which might contribute to trastuzumab resistance, while the underlying mechanisms remain to be further investigated.
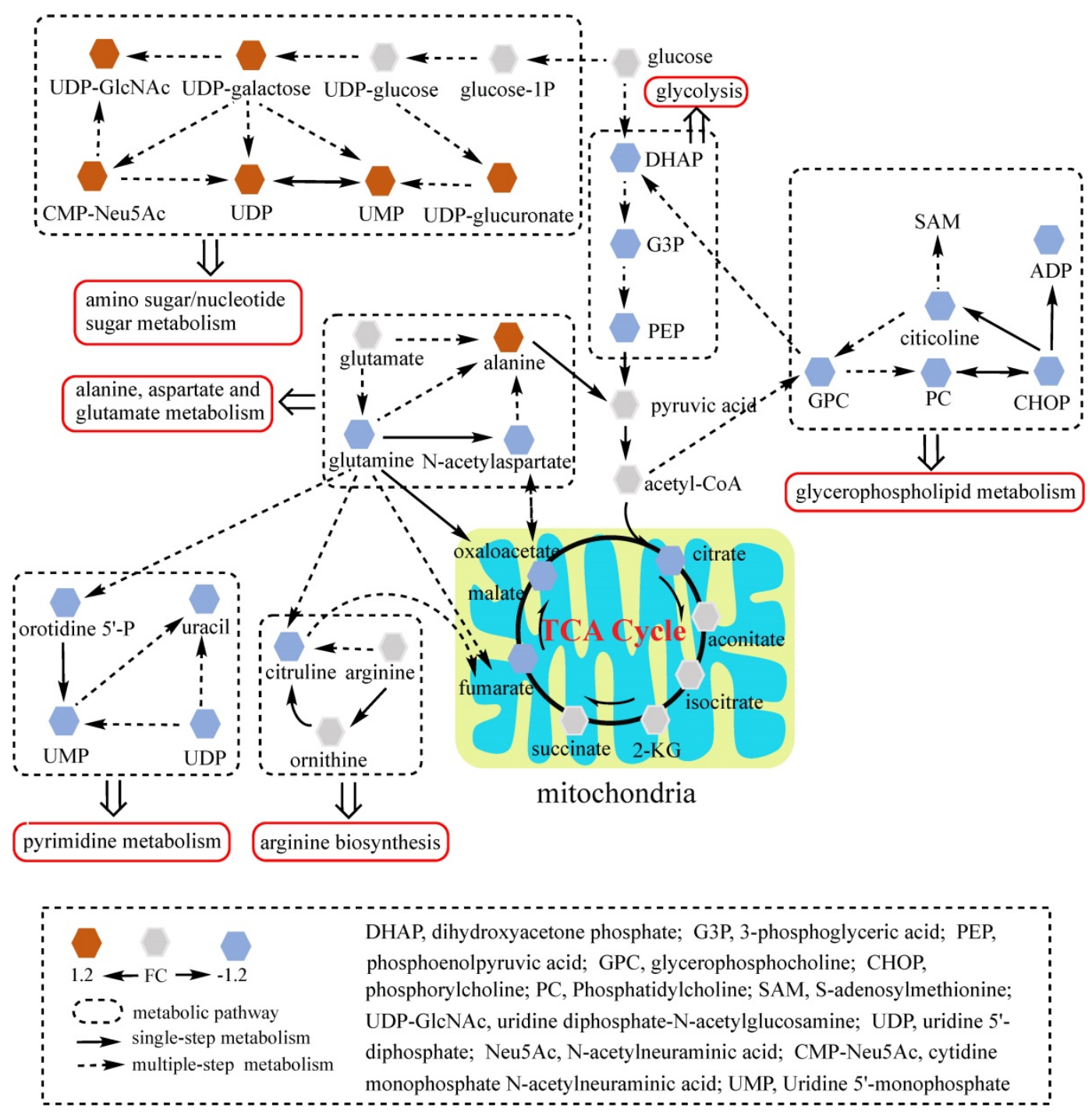

Figure 6. Network analysis of significantly changed metabolic pathways. Seven pathways disturbed in MKN45R/DGATA6 cells were analyzed. Each hexagon represents a metabolite, brown and blue ones represent 1.2 fold increase or decrease in MKN45R/AGATA6 cells respectively. Dashed box indicates different metabolic pathways. Solid arrows show single-step metabolism, and dotted arrows show multiple-step metabolism. 


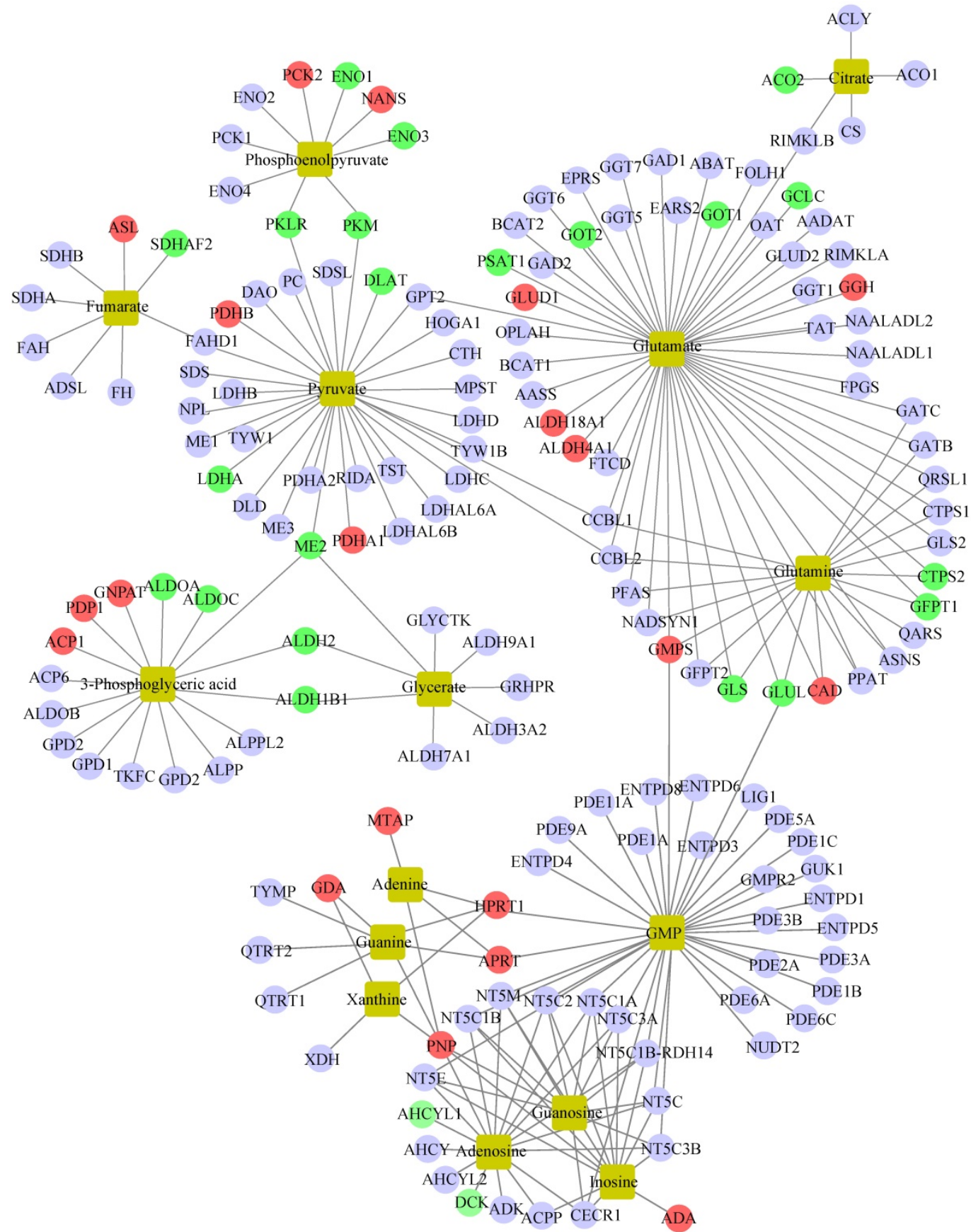

Figure 7. Network analysis of differential metabolites and activated transcriptional factors based on the OmicsNet. Red and green nodes represent up- and down- regulated transcriptional factors, respectively. Light blue nodes indicate transcriptional factors with no significant changes in NCI N87R/ $\triangle$ GATA6 cells compared to NCI N87R. Yellow nodes represent increased and decreased metabolites respectively. Lines represent interaction between transcription factors and metabolites.

Nucleotide metabolism is a key pathway that generates purine and pyrimidine molecules for DNA replication and synthesis. It is well known that enhanced nucleotide metabolism contributes to growth of tumors [38]. Pyrimidines, along with purine metabolism, are two avenues of nucleotide metabolism that produce metabolites including adenine, adenosine, inosine and guanosine monophosphate (GMP), etc. The present study showed that GATA6 knockout led to suppressed purine metabolism since relevant metabolites including adenine, adenosine, xanthine, guanosine, inosine, glutamine, guanine and orotidine-5P were sharply decreased in NCI N87R/ $\triangle$ GATA6 cells. In MKN45R/ $\triangle$ GATA6 cells, pyrimidine metabolism was found to be inhibited. It was widely accepted that glutamine is one of the important precursors for de novo nucleotides synthesis as it provides nitrogen required for purine and pyrimidine synthesis [39]. GLUL, which catalyzes the ATP-dependent conversion of glutamate and ammonia to glutamine, 
was dramatically downregulated upon GATA6 knockout in our study. A study reported that high expression of GLUL affects cellular response to irradiation in radiation-resistant cells and facilitates growth of cancer cells [40]. Thus, it might suggest that nucleotide metabolism mediated by GATA6 also contributes to trastuzumab resistance in gastric cancer cells.

A

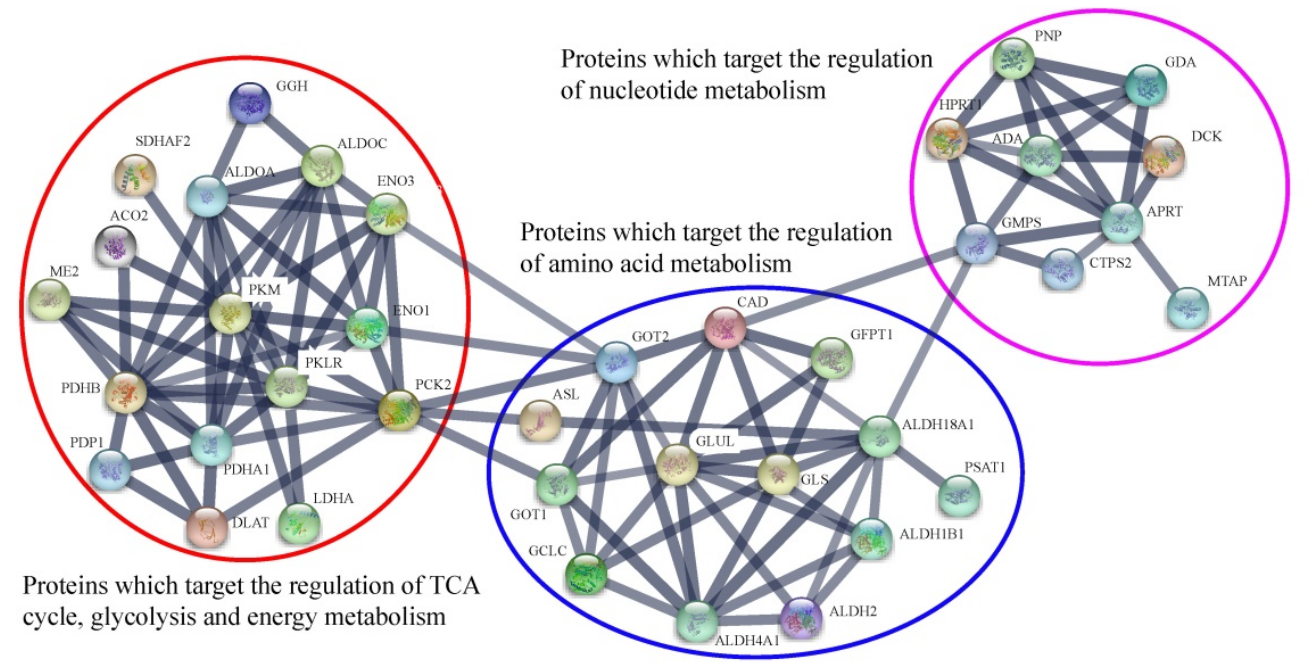

B

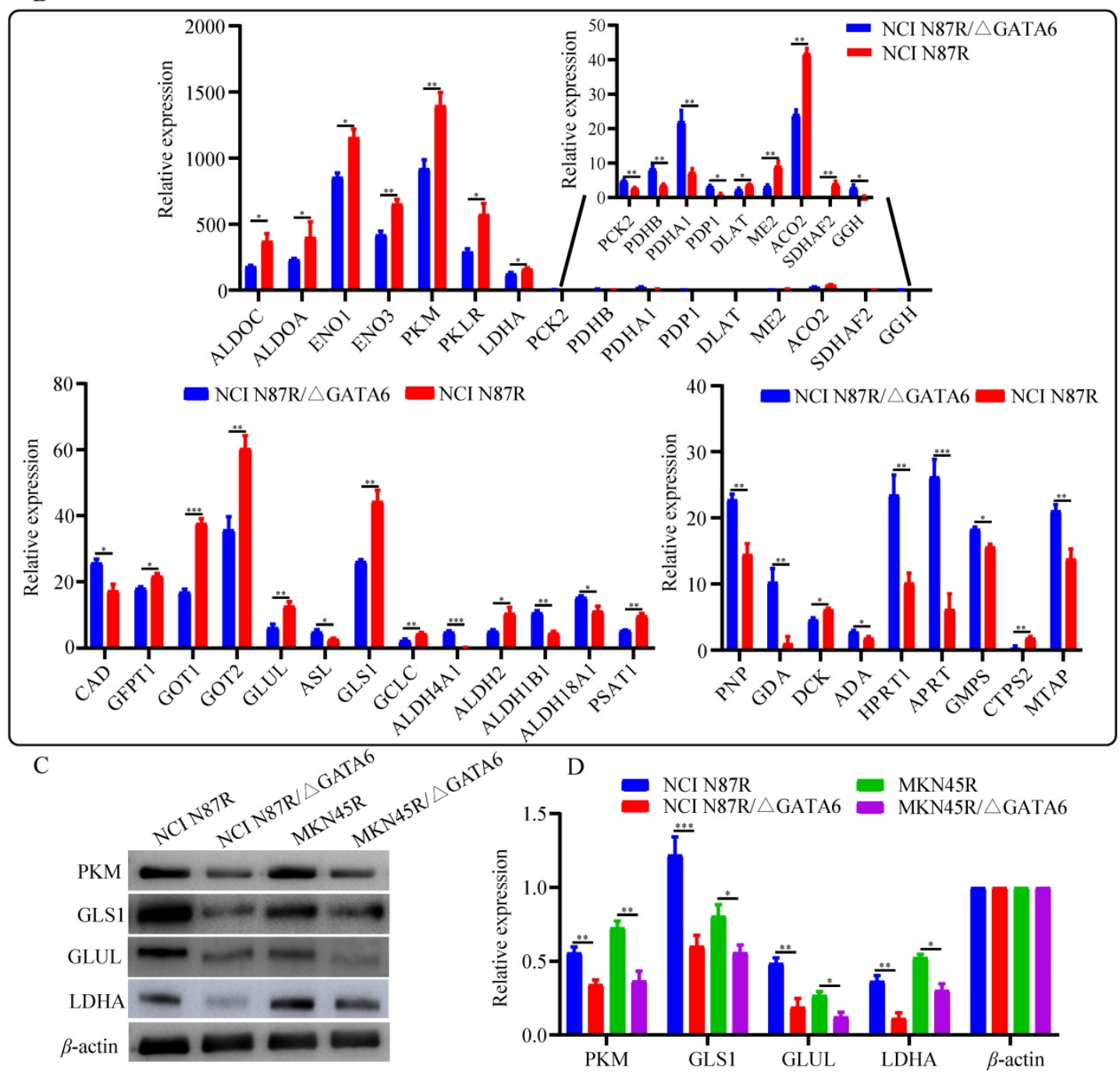

Figure 8. Bioinformatics and quantification analysis of activated transcription factors. (A) Network analysis of activated transcription factors done with Cytoscape software. (B) Quantification of activated transcription factors by mass spectrometry. (C) PKM, GLS1, GLUL and LDHA detected using western blot with $\beta$-actin as loading control. (D) Quantification of western blot signals in two groups of cell lines. Three independent biological replicates are shown as mean \pm SEM. ${ }^{*} p<0.05$, ${ }^{* * *} p<0.01$, ${ }^{* *} p<0.001$ vs. controls. 
In conclusion, our study demonstrated that GATA6 is involved in TCA cycle, glycometabolism, amino acid and nucleotide metabolism, thereby leading to reprogramming in the metabolism and promoting trastuzumab resistance in gastric cancer cells. Recovering the abnormal metabolism of GATA6 in gastric cancer cells could be a potential therapeutic strategy for dealing with trastuzumab resistance.

\section{Abbreviations}

3-PG: 3-phosphoglyceric acid; ACLY: ATP-citrate synthase; ACO1: cytoplasmic aconitate hydratase; ACO2: aconitate hydratase; ACP1: low molecular weight phosphotyrosine protein phosphatase; ADA: adenosine deaminase; ADSL: adenylosuccinate lyase; AGC: automatic gain control; AHCYL1: S-adenosylhomocysteine hydrolase-like protein 1; ALDH1B1: aldehyde dehydrogenase X; ALDH18A1: delta-1-pyrroline-5-carboxylate synthase; ALDH2: aldehyde dehydrogenase; ALDH4A1: delta1-pyrroline-5-carboxylate dehydrogenase; ALDOA/C: fructose-bisphosphate aldolase A/C; APRT: adenine phosphoribosyltransferase; ASL: argininosuccinate lyase; ATP: adenosine triphosphate; CAD: cadherin-1; CHOP: phosphorylcholine; CMP-Neu5Ac: cytidine monophosphate $\mathrm{N}$-acetylneuraminic acid; CS: citrate synthase; CTPs2: CTP synthase 2; DCK: deoxycytidine kinase; DHAP: dihydroxyacetone phosphate; DLAT: dihydrolipoyllysine-residue acetyltransferase component of pyruvate dehydrogenase complex; ENO1/2/3: alpha/gamma/beta-enolase; FAH: fumarylacetoacetase; FH: fumarate hydratase; G3P: 3-phosphoglyceric acid; GAD: glutamate decarboxylase 1; GCLC: glutamate-cysteine ligase catalytic subunit; GFPT1: glutamine-fructose-6phosphate aminotransferase; GGH: gamma-glutamyl hydrolase; GLS: glutaminase kidney isoform; GLUD1: glutamate dehydrogenase 1; GLUL: glutamine synthetase; GMP: guanosine monophosphoric acid; GMPS: GMP synthase; GNPAT: dihydroxyacetone phosphate acyltransferase; GOT: aspartate aminotransferase; GPC: glycerophosphocholine; HPRT1: hypoxanthine-guanine phosphoribosyltransferase; LDHA: L-lactate dehydrogenase A; NANS: sialic acid synthase; NCE: normalized collisional energy; ME2: NAD-dependent malic enzyme; MTAP: S-methyl-5'thioadenosine phosphorylase; OPLS-DA: orthogonal partial least square discriminant analysis; PC: phosphatidylcholine; PCA: principal component analysis; PCK2: phosphoenolpyruvate carboxykinase; PDHA1: pyruvate dehydrogenase E1 component subunit alpha; PDHB: pyruvate dehydrogenase E1 component subunit beta; PDP1: pyruvate dehydrogenase-phosphatase 1; PEP: phosphoenolpyruvic acid; PKLR: pyruvate kinase PKLR; PKM: pyruvate kinase PKM; PNP: purine nucleoside phosphorylase; PSAT1: phosphoserine aminotransferase; SAM: S-adenosylmethionine; SDHA: succinate dehydrogenase flavoprotein subunit; SDHB: succinate dehydrogenase iron-sulfur subunit; SDHAF2: succinate dehydrogenase assembly factor 2; TCA cycle: tricarboxylic acid cycle; UDP: uridine 5'-diphosphate; UDP-GlcNAc: uridine diphosphate$\mathrm{N}$-acetylglucosamine; UDP-Neu5Ac: N-acetylneuraminic acid; UMP: uridine 5'-monophosphate; VIP: variable importance in the projection.

\section{Supplementary Material}

Supplementary figures and tables. http://www.medsci.org/v17p3146s1.pdf

\section{Acknowledgements}

This research was funded by the applied basic research programs of science and technology of Sichuan, China (2019YJ0378), the project of science and technology of Nanchong, China (18SXHZ0402, 19SXHZ0298), and the research fund for doctoral program of North Sichuan Medical College (CBY17-QD05). Data acquisition of UPLC Q-Exactive Focus Orbitrap mass spectrometry was accomplished by Biotree Biotech Co., Ltd. (Shanghai, China).

\section{Competing Interests}

The authors have declared that no competing interest exists.

\section{References}

1. Siegel RL, Miller KD, Jemal A. Cancer statistics, 2020. CA Cancer J Clin. 2020; 70:7-30.

2. Akiyama $\mathrm{T}$, Sudo $\mathrm{C}$, Ogawara $\mathrm{H}$, et al. The product of the human c-erbB-2 gene: a 185-kilodalton glycoprotein with tyrosine kinase activity. Science. 1986; 232:1644-1646.

3. Vernieri $\mathrm{C}$, Milano $\mathrm{M}$, Brambilla $\mathrm{M}$, et al. Resistance mechanisms to anti-HER2 therapies in HER2-positive breast cancer: Current knowledge, new research directions and therapeutic perspectives. Crit Rev Oncol Hematol. 2019; 139:53-66.

4. Chen R, Lai LA, Sullivan Y, et al. Disrupting glutamine metabolic pathways to sensitize gemcitabine-resistant pancreatic cancer. Sci Rep. 2017; 7:7950.

5. Zhou Y, Wang K, Zhou Y, et al. HEATR1 deficiency promotes pancreatic cancer proliferation and gemcitabine resistance by up-regulating Nrf2 signaling. Redox Biol. 2020; 29:101390.

6. Liu W, Yuan J, Liu Z, et al. Label-free quantitative proteomics combined with biological validation reveals activation of $\mathrm{Wnt} / \beta$-catenin pathway contributing to trastuzumab resistance in gastric cancer. Int J Mol Sci. 2018; 19:1981.

7. Sulahian R, Casey F, Shen J, et al. An integrative analysis reveals functional targets of GATA6 transcriptional regulation in gastric cancer. Oncogene. 2014; 33:5637-5648.

8. Song Y, Tian T, Fu X, et al. GATA6 is overexpressed in breast cancer and promotes breast cancer cell epithelial-mesenchymal transition by upregulating slug expression. Exp Mol Pathol. 2015; 99:617-627.

9. Lin L, Bass AJ, Lockwood WW, et al. Activation of GATA binding protein 6 (GATA6) sustains oncogenic lineage-survival in esophageal adenocarcinoma. Proc Natl Acad Sci. 2012; 109:4251-4256.

10. Kamnasaran D, Qian B, Hawkins C, et al. GATA6 is an astrocytoma tumor suppressor gene identified by gene trapping of mouse glioma model. Proc Natl Acad Sci. 2007; 104:8053-8058.

11. Chang J, Wang Y, Zhang F, et al. Proteomic study of transcription factors in trastuzumab-resistant gastric cancer based on liquid chromatography-mass spectrometry technique. Chinese J Anal Chem. 2019; 47:1035-1044. 
12. Liu W, Yuan J, Chang J. Label-free quantitative proteomics for investigation of signaling pathways of GATA6 regulating trastuzumab resistance in gastric cancer cells. Chinese J Anal Chem. 2020; 48:187-196.

13. Shi Y, Wang Y, Huang $\mathrm{W}$, et al. Integration of metabolomics and transcriptomics to reveal metabolic characteristics and key targets associated with cisplatin resistance in non-small cell lung cancer. J Proteome Res. 2019; 18:3259-3267.

14. Liu W, Wang Q, Chang J. Global metabolomic profiling of trastuzumab resistant gastric cancer cells reveals major metabolic pathways and metabolic signatures based on UHPLC-Q Exactive-MS/MS. RSC Adv. 2019; 9:41192-41208

15. Smith CA, Want EJ, O'Maille G, et al. XCMS: processing mass spectrometry data for metabolite profiling using nonlinear peak alignment, matching, and identification. Anal Chem. 2006; 78:779-787.

16. Bijlsma S, Bobeldijk L, Verheij ER, et al. Large-scale human metabolomics studies: a strategy for data (pre-) processing and validation. Anal Chem. 2006; 78:567-574.

17. Chong J, Soufan $\mathrm{O}, \mathrm{Li} \mathrm{C}$, et al. MetaboAnalyst 4.0: towards more transparent and integrative metabolomics analysis. Nucl Acids Res. 2018; 46:486-494.

18. Zhou G, Xia J. Using OmicsNet for network integration and 3D visualization. Curr Protoc Bioinformatics. 2019; 65: e69.

19. Szklarczyk D, Gable AL, Lyon D, et al. STRING v11: protein-protein association networks with increased coverage, supporting functional discovery in genome-wide experimental datasets. Nucl Acids Res. 2019; 47: 607-613.

20. Anderson NM, Mucka P, Kern JG, et al. The emerging role and targetability of the TCA cycle in cancer metabolism. Protein Cell. 2018; 9:216-237.

21. Ren J, Seth P, Clish CB, et al. Knockdown of malic enzyme 2 suppresses lung tumor growth, induces differentiation and impacts $\mathrm{PI}_{3} \mathrm{~K} / \mathrm{AKT}$ signaling. Sci Rep. 2014; 4:5414.

22. Sarfraz I, Rasul A, Hussain G, et al. Malic enzyme 2 as a potential therapeutic drug target for cancer. IUBMB Life. 2018; 70:1076-1083.

23. Pavlova NN, Thompson CB. The emerging hallmarks of cancer metabolism. Cell Metab. 2016; 23:27-47.

24. Zhao H, Duan Q, Zhang Z, et al. Up-regulation of glycolysis promotes the stemness and EMT phenotypes in gemcitabine-resistant pancreatic cancer cells. J Cell Mol Med. 2017; 21:2055-2067.

25. Zhao $\mathrm{Y}$, Zhou M, Liu H, et al. Upregulation of lactate dehydrogenase a by ErbB2 through heat shock factor 1 promotes breast cancer cell glycolysis and growth. Oncogene. 2009; 28:3689-3701.

26. Fantin VR, St-Pierre J, Leder P. Attenuation of LDH-A expression uncovers a link between glycolysis, mitochondrial physiology, and tumor maintenance. Cancer Cell. 2006; 9:425-434.

27. Wang Y, Fan S, Lu J, et al. GLUL promotes cell proliferation in breast cancer. J Cell Biochem. 2017; 118:2018-2025.

28. Cheyne RW, Trembleau L, McLaughlin A, et al. Changes in 2-fluoro-2deoxy-D-glucose incorporation, hexokinase activity and lactate production by breast cancer cells responding to treatment with the anti-HER-2 antibody trastuzumab. Nucl Med Biol. 2011; 38:339-346.

29. Wei Tan H, Ning Leung CO, Shuen Chan KK, et al. Deregulated GATA6 modulates stem cell-like properties and metabolic phenotype in hepatocellular carcinoma. Int J Cancer. 2019; 145:1860-1873.

30. Miao $P$, Sheng $S$, Sun $X$, et al. Lactate dehydrogenase A in cancer: a promising target for diagnosis and therapy. IUBMB Life. 2013; 65:904-910.

31. Zhou M, Zhao Y, Ding Y, et al. Warburg effect in chemosensitivity: targeting lactate dehydrogenase-A re-sensitizes taxol-resistant cancer cells to taxol. Mol Cancer. 2010; 9:33

32. Lukey MJ, Katt WP, Cerione RA. Targeting amino acid metabolism for cancer therapy. Drug Discov Today. 2017; 22:796-804.

33. Altman BJ, Stine ZE, Dang CV. From Krebs to clinic: glutamine metabolism to cancer therapy. Nat Rev Cancer. 2016; 16:619-634.

34. Bott AJ, Maimouni S, Zong W. The pleiotropic effects of glutamine metabolism in cancer. Cancers. 2019, 11:770.

35. Thornburg JM, Nelson KK, Clem BF, et al. Targeting aspartate aminotransferase in breast cancer. Breast Cancer Res. 2008; 10: 84.

36. Hong $\mathrm{C}$, Zheng J, Li X. Inhibition of GOT1 sensitizes colorectal cancer cells to 5-fluorouracil. Cancer Chemother Pharmacol. 2017; 79:835-840.

37. Fu A, Yu Z, Song $\mathrm{Y}$, et al. Silencing of glutaminase 1 re-sensitizes taxol-resistant breast cancer cells to taxol. Mol Med Rep. 2015; 11:4727-4733.

38. Santana-Codina N, Roeth AA, Zhang Y, et al. Oncogenic KRAS supports pancreatic cancer through regulation of nucleotide synthesis. Nat Commun. 2018; 9:4945.

39. Cory JG, Cory AH. Critical roles of glutamine as nitrogen donors in purine and pyrimidine nucleotide synthesis: Asparaginase treatment in childhood acute lymphoblastic leukemia. In vivo. 2006; 20:587-589.

40. Peng Y, Fu S, Hu W, et al. Glutamine synthetase facilitates cancer cells to recover from irradiation-induced G2/M arrest. Cancer Biol Ther. 2020; 21:43-51. 